This is a post-peer-review, pre-copyedit version of an article published in The International Journal of Advanced Manufacturing Technology. The final authenticated version is available online at https://doi.org/10.1007/s00170-019-04443-1.

\title{
A new method to estimate the residual stresses in additive manufacturing characterized by point heat source
}

Li Sun ${ }^{1}$, Xiaobo Ren ${ }^{2}$, Jianying $\mathrm{He}^{1}$, Jim Stian Olsen ${ }^{1}$, Sakari Pallaspuro ${ }^{3}$, Zhiliang Zhang ${ }^{1 *}$

${ }^{1}$ Department of Structural Engineering, Norwegian University of Science and Technology, Trondheim 7491, Norway

${ }^{2}$ SINTEF Industry, Trondheim 7456, Norway

${ }^{3}$ Materials and Mechanical Engineering, Centre for Advanced Steels Research, University of Oulu, Oulu 90570, Finland

${ }^{*}$ Corresponding author: Zhiliang Zhang; E-mail address: zhiliang.zhang@ntnu.no;

\begin{abstract}
Residual stress in additive manufacturing (AM) is one of the key challenges in terms of structural integrity and the finish quality of printed components. Estimating the distribution of residual stresses in additively manufactured components is complex and computationally expensive with full scale thermomechanical FE analysis. In this study, a point heat source is utilized to predict the thermal field and residual stress distribution during the manufacturing processes. Numerical results show that the residual stress at a single material point can be expressed as a function of its spatial position and the peak nodal temperature it has experienced during thermal cycles. The distribution of residual stress can be divided into three segments according to the peak nodal temperature. The peak nodal temperature only depends on the heat flux and the distance to the point heat source center. A semi-analytical approach to predict the peak nodal temperature and residual stresses, once the heat flux is known, is proposed. The proposed approach is further validated by a numerical case study, and a very good agreement has been achieved. Compared to traditional thermo-mechanical FE analysis of additive manufacturing, the proposed method significantly improves the computational efficiency, showing great potential for prediction of residual stresses and distortion.
\end{abstract}

Keywords: Point heat source; Residual stress; Peak nodal temperature; Additive manufacturing;

Nomenclature

$d \quad$ Distance to point heat source center

E $\quad$ Young's modulus

$q \quad$ Heat flux

a Radius of point heat source

$\mathrm{R} \quad$ Radius of axisymmetric model 
$\mathrm{H} \quad$ Height of axisymmetric model

$T_{p} \quad$ Peak temperature the node has experienced during thermal cycles

$T_{m} \quad$ Maximum temperature the model has experienced during thermal cycles

$T_{r} \quad$ Room temperature

$T_{m e l} \quad$ Melting temperature

$T_{e, 1} \quad$ First critical temperature in three-segment equivalent residual stress model

$T_{e, 2} \quad$ Second critical temperature in three-segment equivalent residual stress model

$T_{1,1} \quad$ First critical temperature in three-segment maximum principal residual stress model

$T_{1,2} \quad$ Second critical temperature in three-segment maximum principal residual stress model

$\theta \quad$ The angle to heat surface

$\alpha \quad$ The coefficient of thermal expansion

$\varepsilon_{\text {radiation }}$ Radiation coefficient

$h_{\text {convection }}$ Convection coefficient

$\varepsilon^{*} \quad$ Inherent strain

$\varepsilon_{P} \quad$ Plastic strain

$\varepsilon_{T} \quad$ Thermal plastic strain

$\varepsilon_{X} \quad$ Phase transformation strain

$\sigma_{Y} \quad$ Yield stress

$\sigma^{\text {res }} \quad$ Residual stress

$\sigma_{e}^{r e s} \quad$ Von Mises equivalent residual stress

$\sigma_{1}^{\text {res }} \quad$ Maximum principal residual stress

$\sigma_{e, 1}^{r e s} \quad$ First critical equivalent residual stress

$\sigma_{e, 2}^{r e s} \quad$ Second critical equivalent residual stress

$\sigma_{1,1}^{r e s} \quad$ First critical maximum principal residual stress

$\sigma_{1,2}^{\text {res }} \quad$ Second critical maximum principal residual stress 


\section{Introduction}

Additive manufacturing (AM) or 3D-printing has attracted wide attention over the past years due to its advantages, such as design freedom and short production cycles [90]. Most AM technologies use powder or wire as a feedstock, which is selectively melted by a focused heat source and consolidated in subsequent cooling to form a part layer by layer [1, 18]. Residual stresses will inevitably occur in printed components due to non-uniform material expansion and contraction during the cyclic thermal conditions. It is known that the residual stresses may lead to part distortion, loss of geometric tolerances, and delamination of layers during depositing, as well as to deterioration of the fatigue performance and fracture resistance of a fabricated part $[2,3]$. Hence, accurate prediction of residual stress is a critical issue for AM, which can serve as guidance for the optimization of the AM processes.

Accurate prediction of residual stresses is challenging due to the coupled effects of the materials' thermo-mechanical behavior, microstructure evolution and the fluid flow of the weld pool [4]. Various analytical and computational approaches have been proposed to solve this problem as computational capability has increased, such as the finite element (FE) method. The sequentially-coupled heat conduction analysis in transient mode followed by elastic-plastic small displacement analysis has been the general approach to numerically model thermal distortion and residual stresses in AM [22]. Fullycoupled analysis, which solves the heat conduction and stress equilibrium equations simultaneously, has been used by some studies [23, 91, 92]. However, for FE methods, the transient attribute and the highly nonlinear material behavior result in high computational cost, which limits the models to small workpieces.

To improve the computational efficiency, the inherent-strain method [25] for the prediction of weld distortion of large-scale structures proposed by Yuan et al. has been adopted to the efficient distortion modeling in AM. In this method, the distortion can be calculated by a known inherent strain without a computationally-intensive thermo-mechanical analysis. Although this method was verified in welding distortion modeling, the application for AM distortion modeling with multiple deposition layers is insufficient. Another method was proposed by Li et al. [24] that imported the local residual stress field calculated in the meso-scale layer hatch model to the macro-part model to predict the part distortion and residual stress. However, for a complex part, it would be very difficult for this method to capture the residual stress field precisely. Y. Yang et al. [93] proposed a semi-analytical thermal model of the SLM process which determines the temperature evolution by a finite number of point heat sources. In the model, the superposition of analytical solutions for point sources which are known in semi-infinite space and complementary numerical/analytical fields to impose the boundary conditions are constructed to solve the thermal problem. However, the model can only be applied to bodies having convex surfaces only and becomes cumbersome for complex geometries. 
Some methods were proposed to improve computational efficiency by simplifying the relationship between the peak nodal temperature and residual stress. Mukherjee et al. [27,94] developed an analytical formula which is a function of linear heat input, substrate stiffness, peak nodal temperature, the coefficient of thermal expansion of the depositing alloy and the Fourier number that manifested a ratio of the rate of heat dissipation to storage, for estimating the maximum distortion. Cheng et al. [28] found that the in-plane shrinkage plastic strains can be determined by the peak nodal temperature and material's softening temperature range. Camilleri et al. [26] found that the peak nodal temperature was the dominate thermal parameter that controls the residual stress. Based on the 2D transient thermal analysis, Camilleri et al. [26] developed a so-called mismatched thermal strain (MTS) algorithm to predict the residual stress in 3D welding simulations. Inspired by Camilleri's research, an efficient engineering FE model was developed, in which the model was divided into a plastic zone and an elastic zone based on the peak nodal temperature. The corresponding thermal load according to the nodal response of the plastic flow was then applied to each individual node for the residual stress prediction by Ding's research [29]. As mentioned above, the peak nodal temperature is critical to the prediction of residual stress. However, the mechanical modeling was still performed in a transient way with high computational costs, and a direct relationship between the peak nodal temperature and the residual stress remains unsolved. Due to the complexity and the need for in-house expertise, such strategies have not so far been widely used in industries.

In this work, a direct and efficient methodology based on point heat source to predict residual stresses is proposed, which can be readily used in an industrial context. In section 2, a 3D point heat source model is established numerically to study the thermal field and residual stress distribution. A series of numerical analyses are performed by varying the scale of heat input while keeping geometric parameters fixed. Detailed information about the derivation of the relationship between the peak nodal temperature and the residual stress is presented and a three-segment residual stresses model is developed in section 3. The peak nodal temperature is then expressed analytically as the function of the heat input and the node spatial position in section 4. A direct function relating the heat input and residual stress is presented. This function for calculating the residual stress is then validated by both the 3D point heat source model and numerical AM model in section 5. The main conclusions are presented in section 6.

\section{The point heat source model}

The point heat source model is a semi-infinite solid with a point heat source on it, which can be treated as a simplified solution for welding processes that involve short time heating and cooling cycles (e.g. spot-welding)[95]. In the finite element method, when the point heat source is small enough relative to the geometry, it can also be called a point heat source model. The point heat source model can be used in modeling of welding with a continuous or moving heat source by integrating the total heating time or 
the deposition path. Many numerical and experimental investigations were carried out to study the residual stresses induced by the point heat source [96-98]. However, a direct analytical solution for estimating the residual stress, combining thermal and mechanical analysis, is not available. The point heat source model is built in this section to study the relationship between the heat input and the residual stress distribution.

\subsection{Numerical procedures}

In this work, an axisymmetric point heat source model has been developed in ABAQUS/Standard Ver. 6.14. The effect of model size (radius $R$ and height $H$ ) on the distribution of residual stresses has been studied first. Fig.1 presents the equivalent residual stress $\sigma_{e}^{\text {res }}$ along the top surface (red dash line) with a heat flux $q$ of $4.5 \times 10^{7} \mathrm{~W} / \mathrm{m}^{2}$ and $R / a=H / a$ ranging from 5 to 20 while keeping the radius of point heat source $a$ fixed. Details of the thermal-mechanical analysis will be introduced in section 3 and 4. It can be seen that there is a large difference between the residual stresses of $R / a=5$ and $R / a=10$, while the distributions of residual stress for $R / a=10$ and $R / a=20$ are very close. In this case, the model can be considered as a point heat source model in a semi-infinite body and the simulation results are independent of $R / a$ when $R / a \geq 10$. In this study, the radius $(R)$ and the height $(H)$ of the model are $50 \mathrm{~mm}$, while the radius of the heat source (a) is $5 \mathrm{~mm}$, i.e. $R / a=H / a=10$.

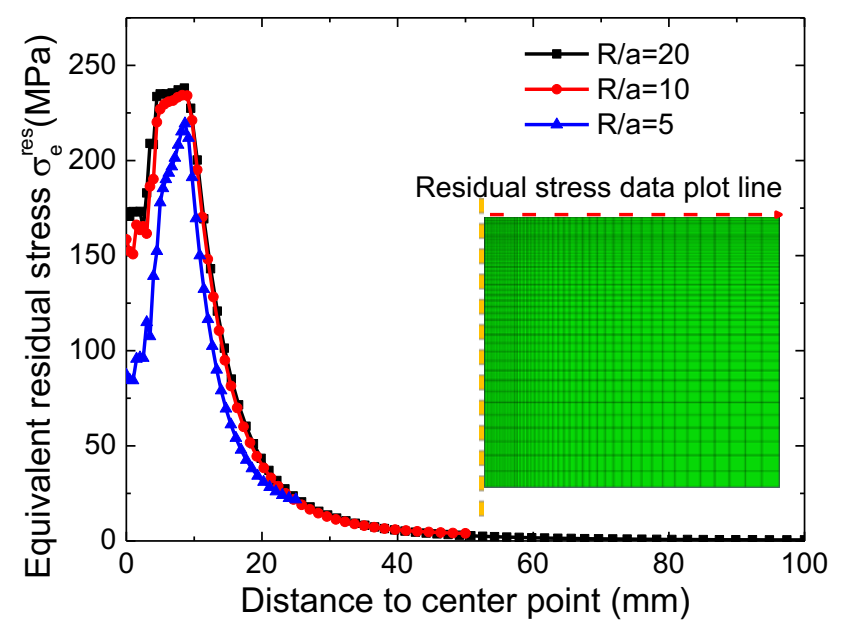

Fig. 1. The effect of model size on the residual stress distribution

The initial ambient temperature was assumed to be $20{ }^{\circ} \mathrm{C}$, and both heating and cooling processes are considered. The effect of thermal cycles (1,2 and 3) on the simulation results have also been studied. Fig.2 presents the equivalent residual stress $\sigma_{e}^{\text {res }}$ and maximum principal residual stress $\sigma_{1}^{\text {res }}$ along the top surface (red dash line) in different thermal cycles. The heat flux inputs are $2.5 \times 10^{7} \mathrm{~W} / \mathrm{m}^{2}$, $2 \times 10^{7} \mathrm{~W} / \mathrm{m}^{2}$ and $2 \times 10^{7} \mathrm{~W} / \mathrm{m}^{2}$ respectively in the three thermal cycles. The number of thermal cycles has a significant effect on the distribution of residual stresses in the area close to the heat source 
and the effect can be ignored. Because the residual stresses are equal to the yield stress as a conservative approach based on the three-segment model which will be introduced in detail in section 3 and 4 . For the rest part, the effect becomes negligible as the residual stresses are mainly dependent on the peak nodal temperature $T_{P}$ which occurs in the first thermal cycle. Hence, the effect of thermal cycles can be ignored and the temperature and residual stress evolution in point heat source model can be determined by way of representing the thermal history with one thermal cycle.

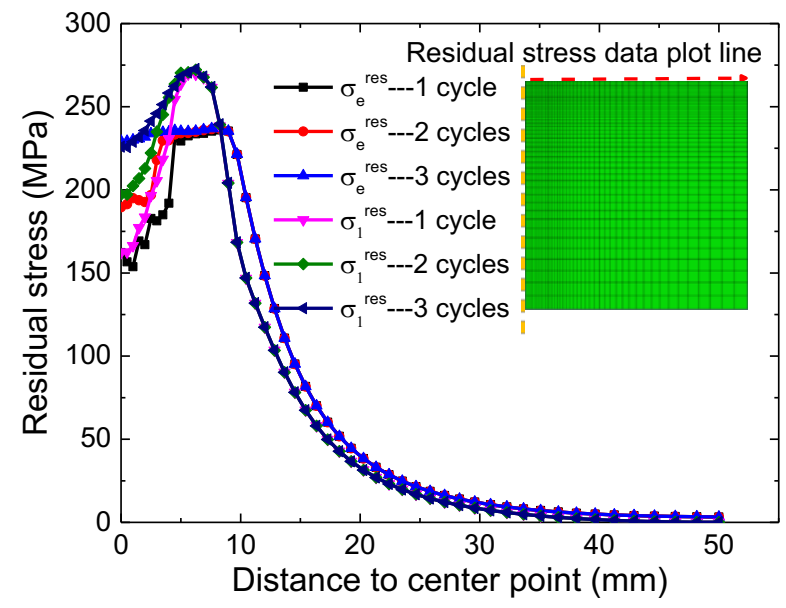

Fig. 2. The effect of thermal cycles on the residual stress distribution

In this work, one thermal cycle consists of heating process flowed by air cooling process. During the heating process, uniform heat will be input through the heat source area. Considering the high deposition speed and the maximum temperature in the welding process or AM, the heating stage lasts only for 2 seconds and $q$ ranges from $2.5 \times 10^{7} \mathrm{~W} / \mathrm{m}^{2}$ to $4.5 \times 10^{7} \mathrm{~W} / \mathrm{m}^{2}$. The surfaces of the model exposed to the air were subjected to convection and radiation heat loss (convective coefficient of $8.5 \mathrm{~W} /\left(\mathrm{m}^{2} \mathrm{~K}\right)$ and radiation coefficient of 0.8 ). While the bottom contacting the support base plate were subjected to conduction loss with a thermal conductivity coefficient of $123 \mathrm{~W} /\left(\mathrm{m}^{2} \mathrm{~K}\right)$ at $20{ }^{\circ} \mathrm{C}[87,88]$, which is modeled by an equivalent convection coefficient $\left(123 \mathrm{~W} / \mathrm{m}^{2} \mathrm{k}\right)[86,87]$ in this work. A sufficiently long waiting time is used in the cooling stage to guarantee the model to be cooled down to the room temperature naturally.

As the heating source radius is $5 \mathrm{~mm}$ and the heating stage lasts for 2 seconds, hence the heat flux density of the point heat source model approximately equal to the heat flux density when the welding speed is 5 $\mathrm{mm} / \mathrm{s}$. In welding and direct deposition additive manufacturing process, the deposition speed is usually $3.5 \mathrm{~mm} / \mathrm{s}$ to $10 \mathrm{~mm} / \mathrm{s}$. The influence is negligible when the speed does not change much. Hence, the heating time $2 \mathrm{~s}$ can be typically used to simulate the welding and additive manufacturing process. Hence, the influence of the heating time is not considered in this work. 


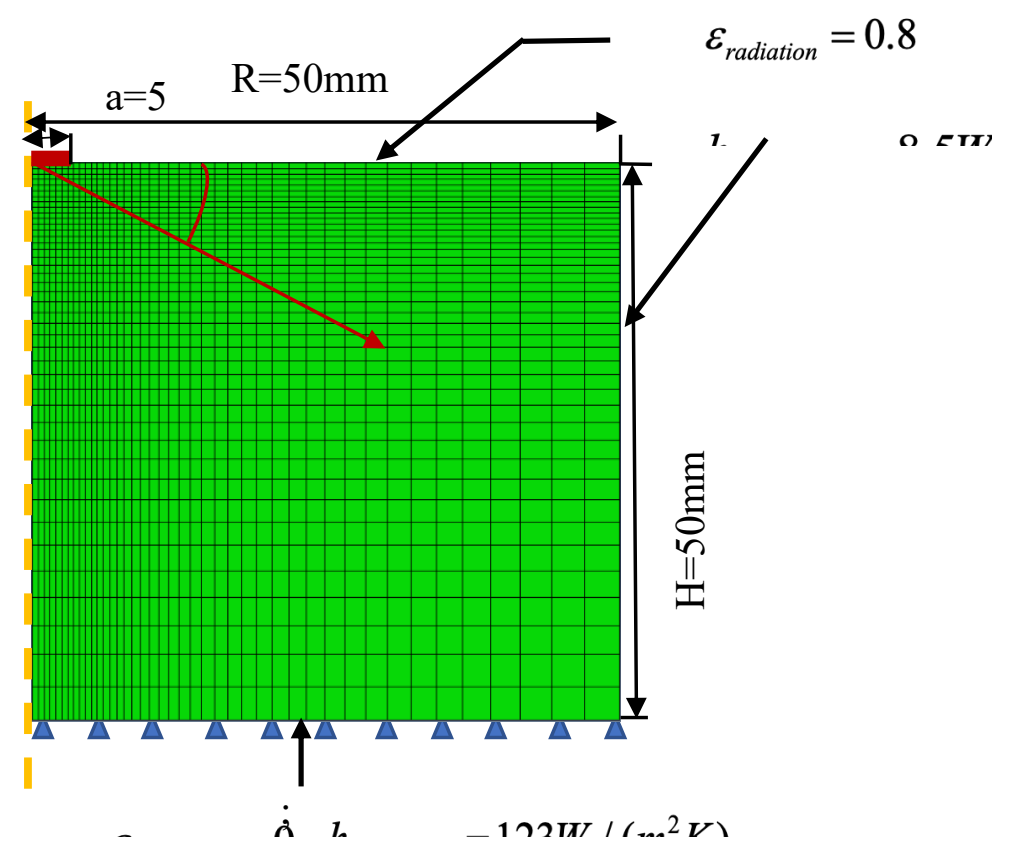

Fig. 3 Mesh of the axisymmetric point heat source model

The four-node axisymmetric thermally coupled quadrilateral, bilinear displacement and temperature element (CAX4T) is selected. The mesh is shown in Fig. 3 as well as the thermal boundary conditions. After the mesh sensitive analysis, the average mesh size near the point heat source used is $0.5 \times 0.5 \mathrm{~mm}$. Relatively coarse mesh is assigned in the remaining part. The axisymmetric boundary condition is applied in the symmetric plane, shown with the yellow dash line, while the bottom is fixed.

\subsection{Material AA2319}

The temperature dependent material properties of aluminum alloy 2319 (AA2319), such as the thermal conductivity coefficient, thermal expansion coefficient, temperature dependent yield stress, etc., are obtained from [84] and are presented in Fig. 4 (a) - (d). Mass density is assumed to be $2823 \mathrm{~kg} / \mathrm{m}^{3}$ and temperature independent. The melting range (the span of temperature from the point at which the crystals first begin to liquefy to the point at which the entire sample is liquid) of AA2319 is $543{ }^{\circ} \mathrm{C}-643{ }^{\circ} \mathrm{C}$. The temperature dependent constitutive relationship of the true stress and the true strain are presented in Fig. 4 (d). The softening effect is not considered in this model. 


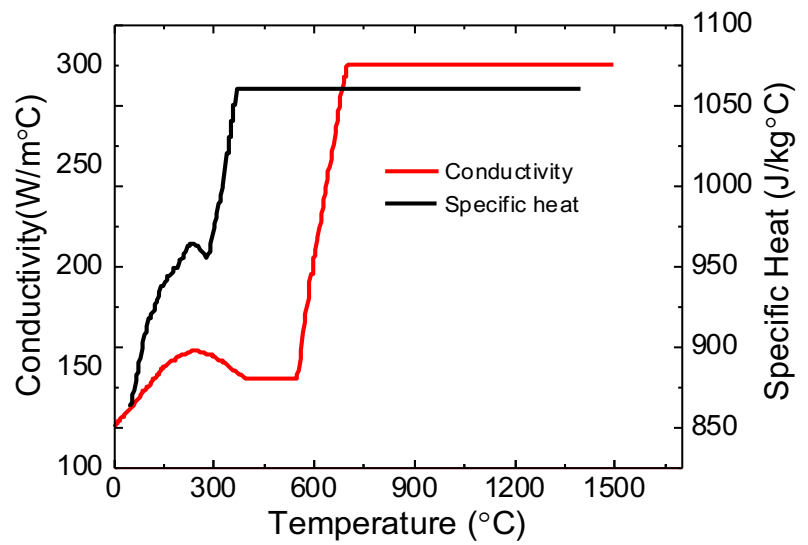

(a)

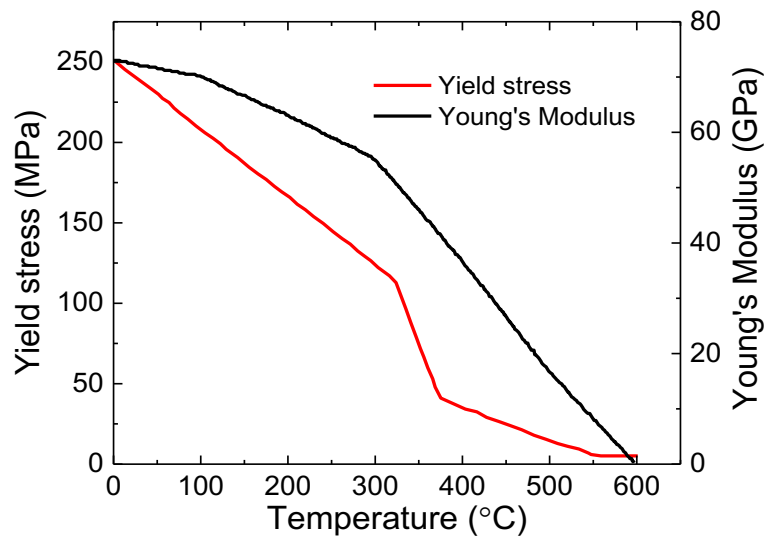

(c)

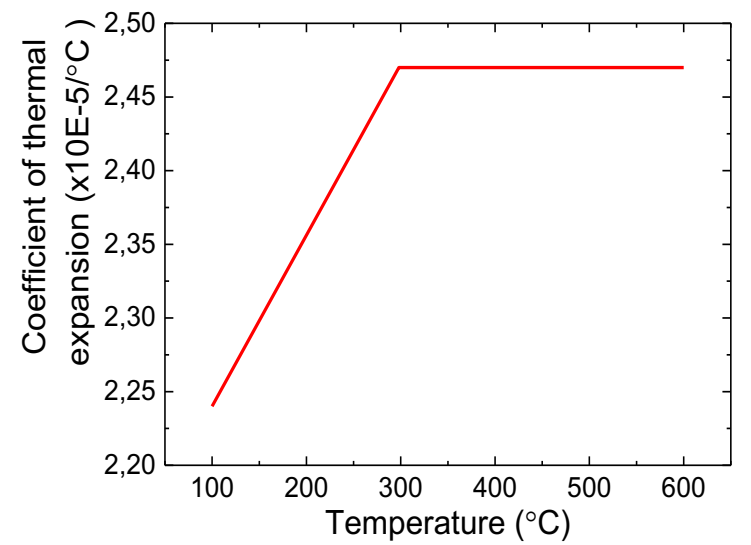

(b)

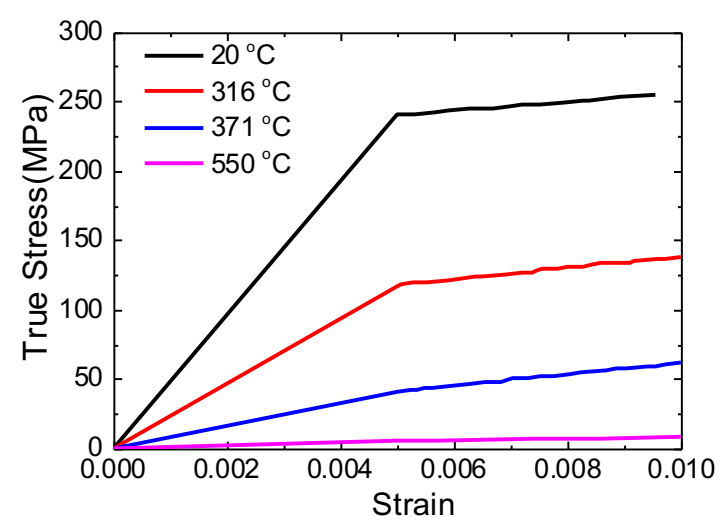

(d)

Fig. 4 Physical properties of AA2319: (a) thermal conductivity and specific heat, (b) coefficient of thermal expansion, (c) Young's modulus and yield strength, and (d) strain-hardening [84].

\section{Three-segment residual stress model}

\subsection{Key parameters affecting residual stresses}

The AM and fusion welding share many of the same physical phenomena, especially those key physical factors governing the formation of residual stresses and distortion. The origins of residual stresses include the spatial temperature gradient, thermal expansion and contraction, and strain compatibility. The spatial temperature gradient in a simplified model that is influenced by the maximum temperature that the whole model experienced $T_{m}$. The thermal expansion and contraction of a material point caused by the localized heating and cooling process depend mainly on the peak nodal temperature, $T_{P}$. The strain compatibility, i.e. uneven distribution of inelastic strains, force equilibrium, and constitutive stress-strain behavior will also affect the residual stress $[8,99]$. It has also been considered that the residual stresses come from the inherent strain $\varepsilon^{*}$. The inherent strain can be described as a combination of the phase transformation strain $\varepsilon_{X}$, the thermal plastic strain $\varepsilon_{T}$ which depends on $T_{P}$ and the plastic strain $\varepsilon_{P}$ which is influenced by $T_{m}$ and the node spatial position $[8,21]$, 


$$
\varepsilon^{*}=\varepsilon_{T}+\varepsilon_{p}+\varepsilon_{X}
$$

The spatial position of a single material point can be represented simply by its polar coordinate parameter $\theta$ and $d$. $\theta$ is the clock-wise angle and $d$ is the radius to the pole, as shown in Fig. 2. In this study, since AA 2319 alloy only shows one phase, $\alpha$-phase in Face Centered Cubic Structure, no phase transformations in the AA 2319 alloy is considered and the precipitation effects are neglected. Correspondingly, the phase transformation strain $\varepsilon_{X}$ is assumed to be zero and canceled out in Eq. (1).

According to the analysis above, the residual stress can be expressed as a function of $\theta, d / a, T_{m}$ and $T_{P}$,

$$
\sigma^{r e s}=f\left(\theta, d / a, T_{m}, T_{p}\right)
$$

The purpose of the following work is to link $T_{P}$ and $T_{m}$ to the heat input $q$ and the nodal spatial coordinates $(d / a$ and $\theta)$ to obtain the final function form for the residual stress prediction,

$$
\sigma^{r e s}=f(\theta, d / a, q)
$$

In this work, only $\sigma_{e}^{\text {res }}$ and $\sigma_{1}^{\text {res }}$ are considered, since $\sigma_{e}^{\text {res }}$ is relevant to plastic yielding while the $\sigma_{1}^{\text {res }}$ can be considered as a prime indicator on fatigue and fracture performance [21]. Similar pattern for other components can be obtained by using the proposed approach.

\subsection{Three-segment equivalent residual stress model}

Fig. 5 shows that $\sigma_{e}^{\text {res }}$ distributes non-uniformly on the whole model after a heating and cooling cycle with a heat flux of $3.5 \times 10^{7} \mathrm{~W} / \mathrm{m}^{2}$. Especially close to the point heat source, the equivalent residual stress is much larger than that of the remaining part far from the center point heat source.

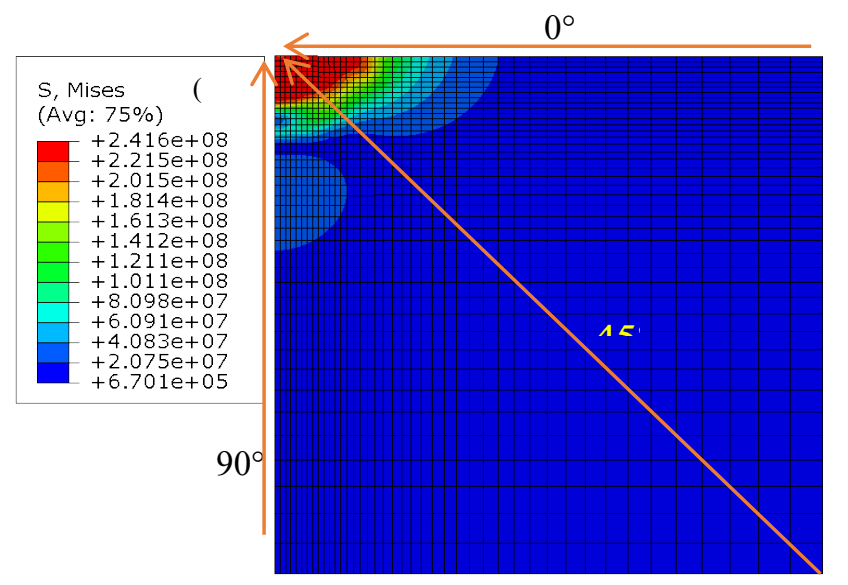

Fig. 5. The equivalent residual stress distribution.

The $\sigma_{e}^{r e s}$ and $T_{P}$ in different directions $\left(\theta=0^{\circ}, 22.5^{\circ}, 45^{\circ}, 67.5^{\circ}, 90^{\circ}\right)$ were extracted. $\sigma_{e}^{r e s}$ is normalized by the yield stress $\left(\sigma_{Y}=243 M P a\right)$ while $T_{P}$ is normalized by the melting temperature $\left(T_{m e l}=643{ }^{\circ} \mathrm{C}\right)$. After the normalization, the corresponding results are plotted in Fig. 6 with $q$ ranging 
from $2.5 \times 10^{7} \mathrm{~W} / \mathrm{m}^{2}$ to $4.5 \times 10^{7} \mathrm{~W} / \mathrm{m}^{2}$. In subfigures, $T_{P} / T_{m e l}$ increases from 0.03 to the maximum, with respect to the nodes from the edge point to the heat source center, as the arrows shown in Fig. 5.

As the subfigures showed, the $\sigma_{e}^{r e s} / \sigma_{y}-T_{p} / T_{\text {mel }}$ curve shape evolves with $\theta$, but $q$ shows very minor effect. That is, at a given angle $\sigma_{e}^{\text {res }}$ is mainly dependent on $T_{P}$. There are four segments divided by the turning points $P_{1}$ to $P_{3}$, which are best visible at $45^{\circ}$ (Fig. $\left.6(\mathrm{c})\right)$. The first segment reaches up to $P_{1}$, and the corresponding zone is named here as the boundary zone. It can be observed that $P_{1}$ is not obvious for $0^{\circ}$ and $22.5^{\circ}$, as $\sigma_{e}^{r e s} / \sigma_{y}$ increases linearly from 0 to 1 . Because the top and right surfaces are free and the boundary effect is small. At $45^{\circ}, 67.5^{\circ}$, and $90^{\circ}$, the bottom surface is fixed and the boundary effect is large, hence points with respect to $P_{1}$ are obvious and the curves are wave-like between the start point and $P_{1}$. And the corresponding values of $\sigma_{e}^{r e s} / \sigma_{y}$ are around $0.08(0.074,0.082$, and 0.082$)$. Therefore, the points with $\sigma_{e}^{r e s} / \sigma_{y}$ equaling to 0.08 in every direction are set as the first critical point, $P_{1}$. In the second segment, i.e. in the transition zone between $P_{1}$ and $P_{2}, \sigma_{e}^{r e s} / \sigma_{y}$ increases sharply with $T_{P} / T_{m e l}$. For $P_{2}$, it is obvious in all directions because the $\sigma_{e}^{r e s} / \sigma_{y}$ corresponding to $P_{2}$ is exactly equal to 1 . The third segment between $P_{2}$ to $P_{3}$ is the so-called yield zone since $\sigma_{e}^{\text {res }}$ is almost constant and equal to the yield stress. In the final segment, i.e. the release zone after $P_{3}, \sigma_{e}^{r e s} / \sigma_{y}$ decreases with $T_{P} / T_{m e l}$, as the material has melted, accompanied by the stresses relief due to the free surface expansion. The effects of stress relief are omitted as a conservative approach and the release zone is merged into the yield zone. Hence, the equivalent residual stress can be divided into three segments, i.e. the boundary zone, the transition zone, and the yield zone. 


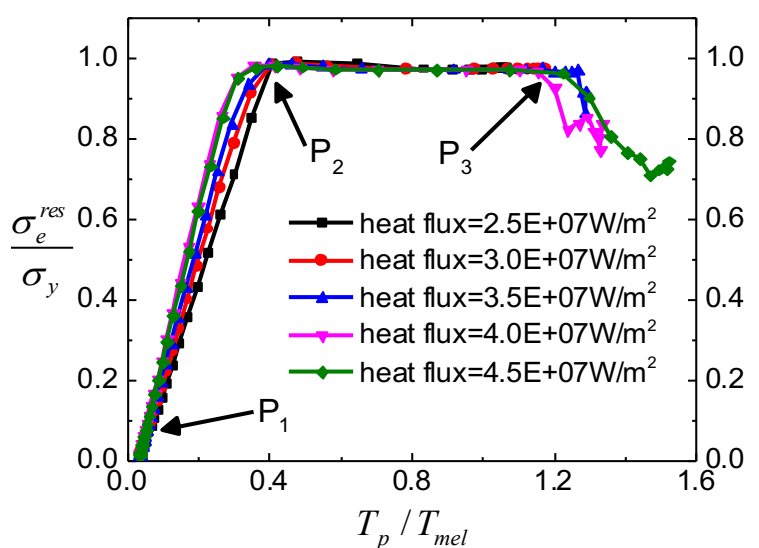

(a)

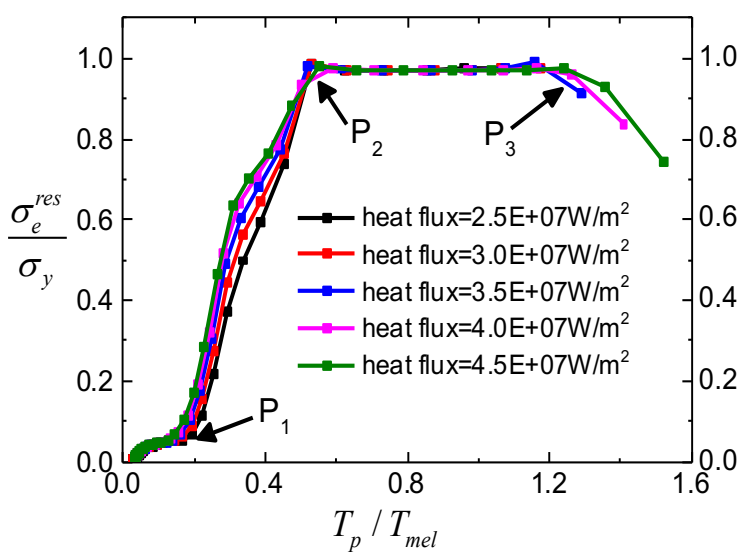

(c)

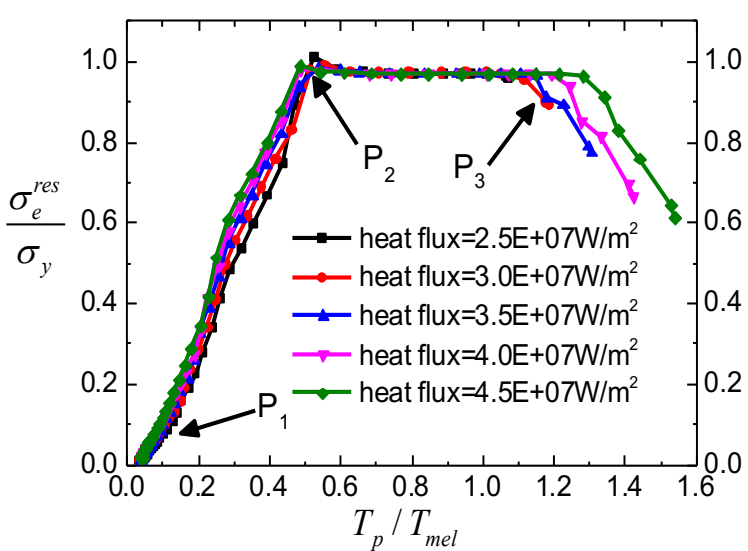

(b)

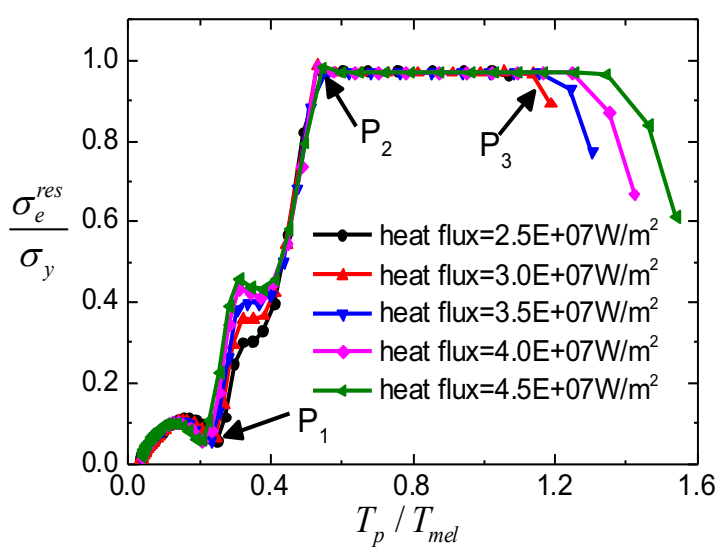

(d)

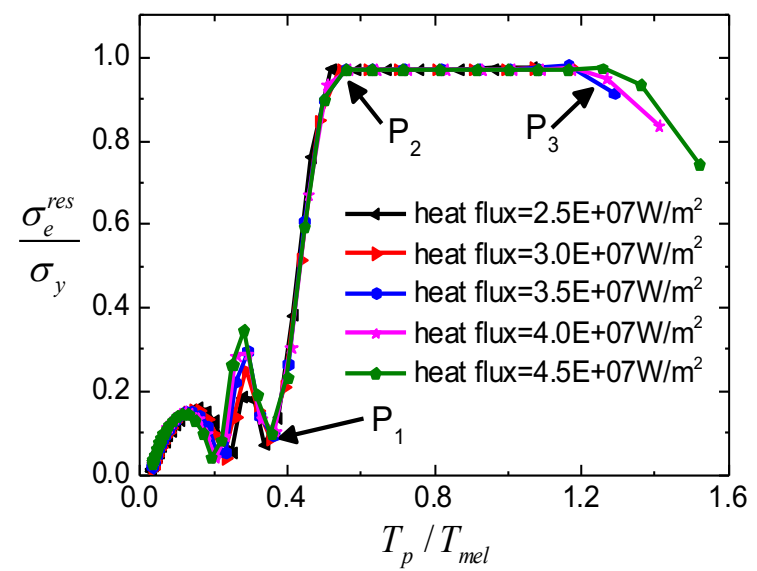

(e)

Fig .6. Normalized equivalent residual stress $\sigma_{e}^{\text {res }} / \sigma_{y}$ versus normalized peak nodal temperature

$$
T_{P} / T_{m e l} \text { : (a) } \theta=0^{\circ} \text {, (b) } \theta=22.5^{\circ} \text {, (c) } \theta=45^{\circ} \text {; (d) } \theta=67.5^{\circ} \text {, and (e) } \theta=90^{\circ} \text {. }
$$

In the three-segment model, $\sigma_{e}^{r e s} / \sigma_{y}$ is simplified to vary linearly with $T_{P} / T_{m e l}$ in the first and second segment, and to be equal to one in the third segment. The three-segment equivalent residual stress model is described in Fig. 7 and the formula for calculating $\sigma_{e}^{r e s}$ is expressed as: 


$$
\frac{\sigma_{e}^{r e s}}{\sigma_{y}}=\left\{\begin{array}{cc}
\frac{\sigma_{e, 1}^{r e s} *\left(T_{p}-T_{r}\right)}{\sigma_{y} *\left(T_{e, 1}-T_{r}\right)} & \left(T_{r} \leq T_{p} \leq T_{e, 1} ; 0^{\circ} \leq \theta \leq 90^{\circ}\right) \\
\frac{\sigma_{e, 1}^{r e s}}{\sigma_{y}}+\frac{\left(T_{e, 1}-T_{p}\right)}{\left(T_{e, 1}-T_{e, 2}\right)} *\left(1-\frac{\sigma_{e, 1}^{r e s}}{\sigma_{y}}\right) & \left(T_{e, 1} \leq T_{p} \leq T_{e, 2} ; 0^{\circ} \leq \theta \leq 90^{\circ}\right) \\
1 & \left(T_{p} \geq T_{e, 2} ; 0^{\circ} \leq \theta \leq 90^{\circ}\right)
\end{array}\right.
$$

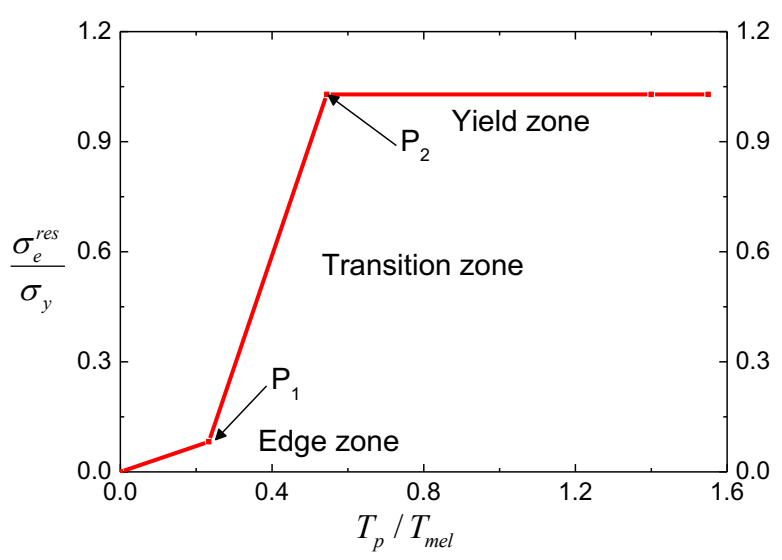

Fig. 7. Three-Segment equivalent residual stress model.

Therefore, if $T_{e, 1} / T_{m e l}, T_{e, 2} / T_{m e l}$, and $\sigma_{e, 1}^{r e s} / \sigma_{y}, \sigma_{e, 2}^{r e s} / \sigma_{y}$ at the turning points $P_{1}$ and $P_{2}$ are known, then the $\sigma_{e}^{r e s}$ distribution can be obtained based on the three-segment model. Since $\sigma_{e, 1}^{r e s} / \sigma_{y}$ is equal to 0.08 and $\sigma_{e, 2}^{r e s} / \sigma_{y}$ is equal to 1 , only $T_{e, 1} / T_{m e l}$ and $T_{e, 2} / T_{m e l}$ need to be determined.

\subsubsection{The normalized first critical temperature $T_{e, 1} / T_{m e l}$}

$T_{e, 1} / T_{m e l}$ in different directions is obtained and plotted against the angle $\theta$ in Fig. 8. As can be seen, $T_{e, 1} / T_{m e l}$ increases with the increase of $\theta$. This is due to that the large the angle is, the more constraint the material subjected to the surrounding cold material. Hence, the large the angle is, the more the residual stress gradient is. For the same equivalent residual stress at $P_{1}$, a smaller distance and corresponding higher $T_{e, 1} / T_{m e l}$ can be expected. The data in Fig. 8 is then fitted by a second-order function:

$$
\frac{T_{e, 1}}{T_{m e l}}=2.95 \times e^{-5} \theta^{2}+3.41 \times e^{-4} \theta+8.29 \times e^{-2}
$$




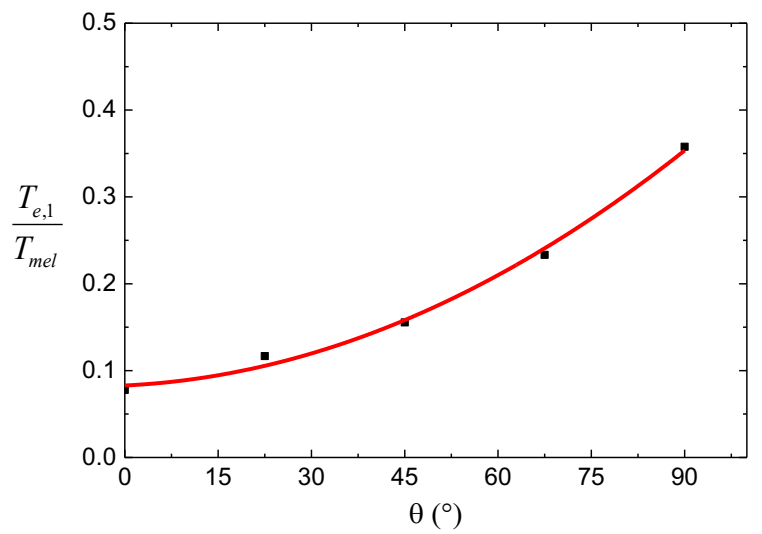

Fig. 8. Normalized first critical temperature $T_{e, 1} / T_{\text {mel }}$ versus angle $\theta$.

\subsubsection{The normalized second critical temperature $T_{e, 2} / T_{m e l}$}

The second critical point divides the model into the yield zone and the transition zone. $T_{e, 2} / T_{m e l}$ mentioned in Fig. 7 is presented in Fig. 9 as a function of the angle $\theta$. It can be seen that $T_{e, 2} / T_{m e l}$ is around 0.51 with small deviations, namely $T_{e, 2}=330{ }^{\circ} \mathrm{C}$. It has been proved in such strategies have not [26] that the maximum thermal strain $\alpha\left(T_{e, 2}-T_{r}\right)$ should exceed two times the yield strain in the heating process if the material yields. Therefore, the temperature corresponding to the yield stress can be calculated by:

$$
2 \frac{\sigma_{y}}{E}=\alpha\left(T_{e, 2}-T_{r}\right)
$$

where the yield stress $\sigma_{y}=243 \mathrm{MPa}$, Young's modulus $E=70 \mathrm{GPa}$, coefficient of thermal expansion $\alpha=2.24 \times 10^{-5} /{ }^{\circ} \mathrm{C}$ and room temperature $T_{r}=20 C^{\circ} . T_{e, 2}$ calculated by Eq. (6) is equal to $330{ }^{\circ} \mathrm{C}$ and $T_{e, 2} / T_{m e l}$ is equal to 0.51 . Hence, it is more convenient to obtain $T_{e, 2} / T_{m e l}$ with known yield stress by Eq. (6).

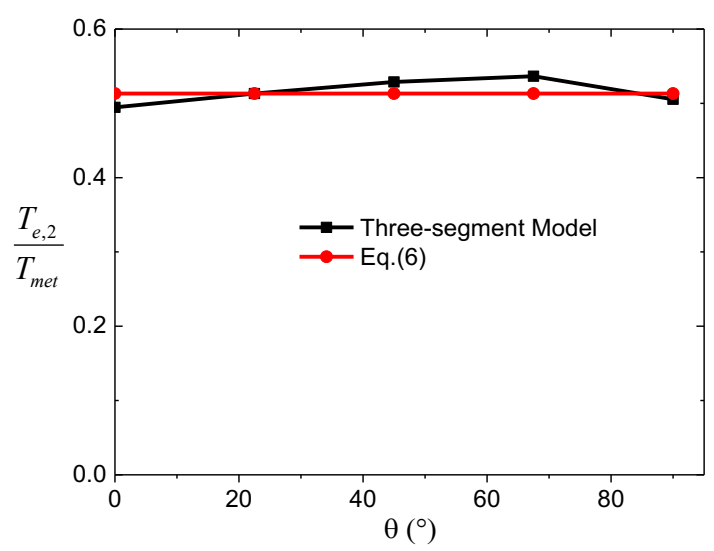

Fig. 9. Normalized second critical temperature $T_{e, 2}$ versus angle $\theta$. 


\subsection{Three-segment maximum principal residual stress model}

The maximum principal stress distribution after a heating and cooling cycle with a heat flux of $3.5 \times 10^{7} \mathrm{~W} / \mathrm{m}^{2}$ has also been studied and presented in Fig. 10. It can be seen that $\sigma_{1}^{\text {res }}$ is much higher in the vicinity of the point heat source than in the part close to the free surface. Similar to the analyses in section 3.2, the normalized maximum principal residual stress $\sigma_{1}^{\text {res }} / \sigma_{y}$ in different directions $\left(\theta=0^{\circ}, 22.5^{\circ}, 45^{\circ}, 67.5^{\circ}, 90^{\circ}\right)$ is derived and plotted against the normalized peak nodal temperature $T_{p} / T_{m e l}$ in Fig. 11 with $q$ ranging from $2.5 \times 10^{7} \mathrm{~W} / \mathrm{m}^{2}$ to $4.5 \times 10^{7} \mathrm{~W} / \mathrm{m}^{2}$.

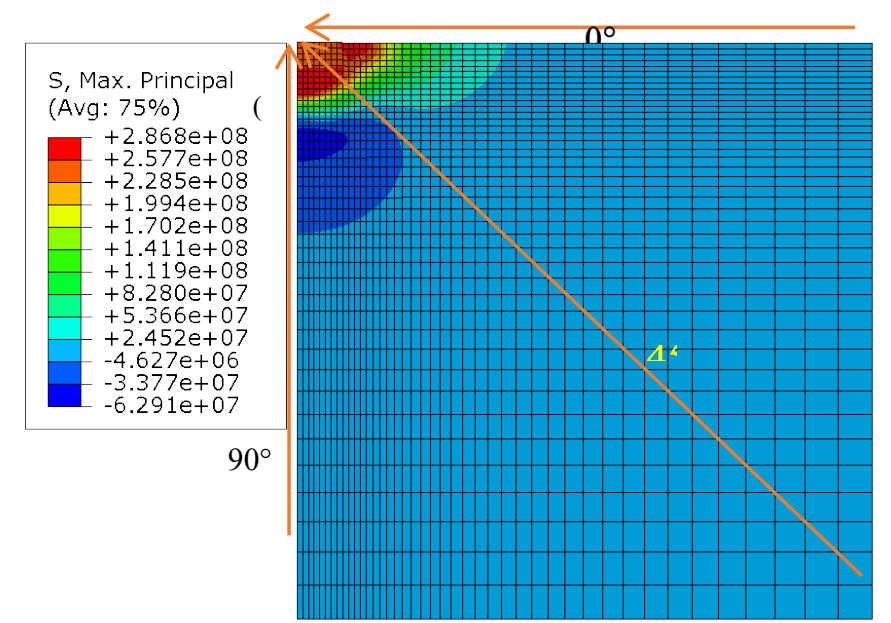

Fig. 10. The maximum principal residual stress distribution.

Similarly to the results in section 3.2, the curves in Fig. 11 can also be divided into 3 segments, namely, the boundary zone, the transition zone, and the yield zone by the turning points $S_{1}$ and $S_{2}$. Different to the definition of the first turning point in section 3.2, the $S_{1}$ separating the boundary zone and the transition zone is defined by the $T_{p} / T_{m e l}$ at 0.23 since $T_{p} / T_{m e l}$ corresponding to the first turning point in Fig. 11 (c) - (e) is almost the same. For the second turning point, it is determined by the value of the $\sigma_{1}^{r e s} / \sigma_{y}$ corresponding to 1 .

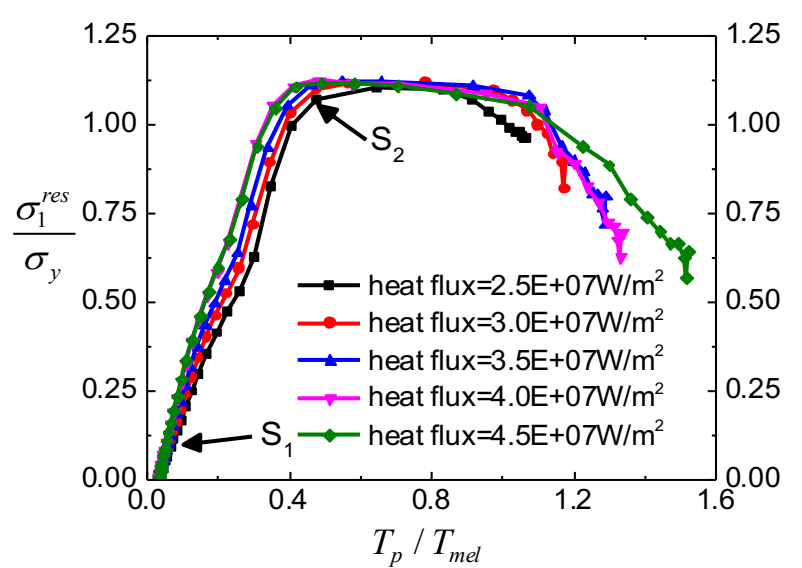

(a)

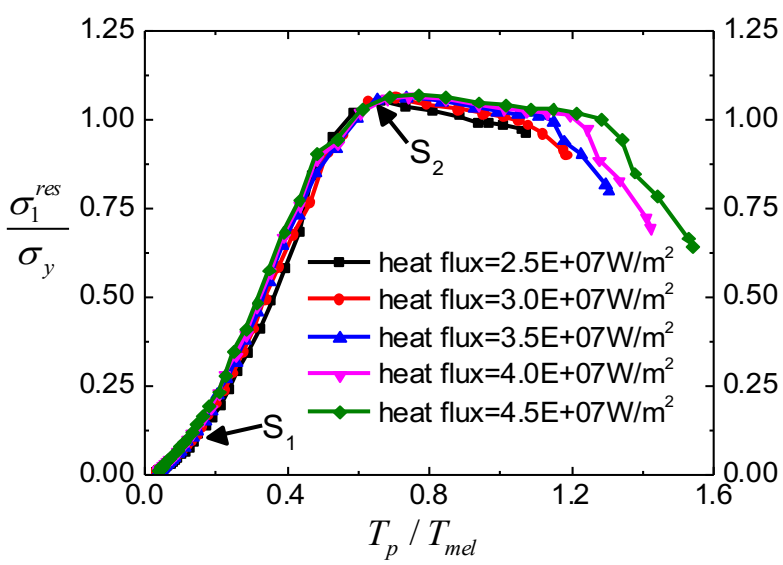

(b) 


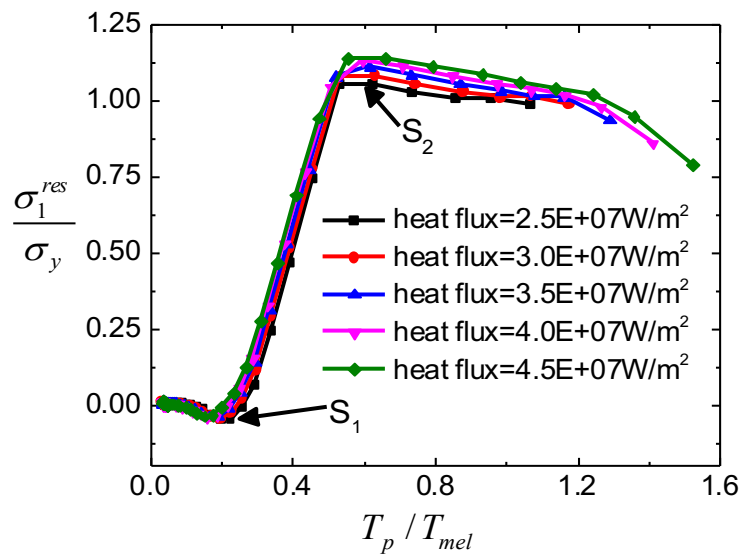

(c)

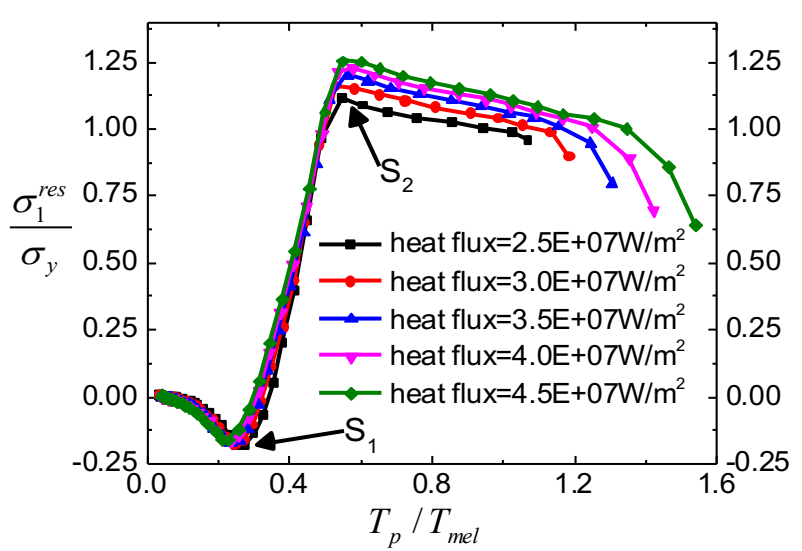

(d)

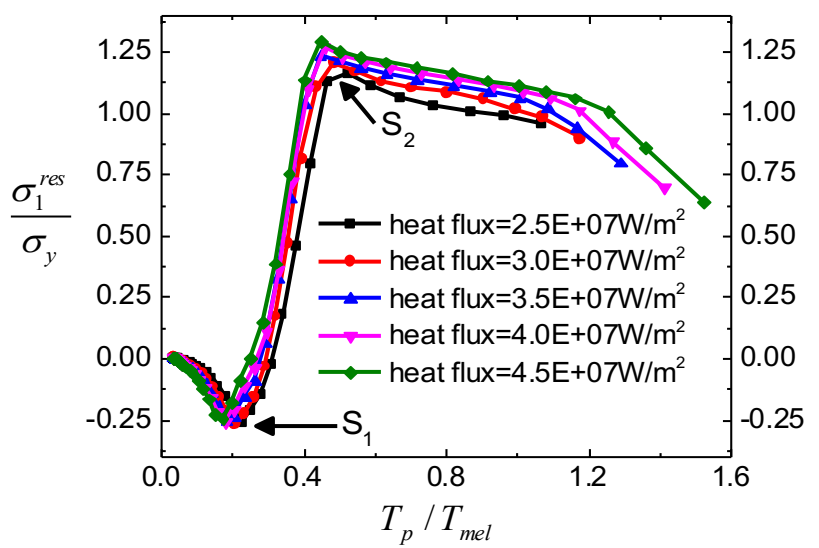

(e)

Fig. 11. Normalized maximum principal residual stress $\sigma_{1}^{\text {res }} / \sigma_{y}$ versus normalized peak nodal temperature $T_{p} / T_{m e l}$ : (a) $\theta=0^{\circ}$, (b) $\theta=22.5^{\circ}$, (c) $\theta=45^{\circ}$; (d) $\theta=67.5^{\circ}$, and (e) $\theta=90^{\circ}$.

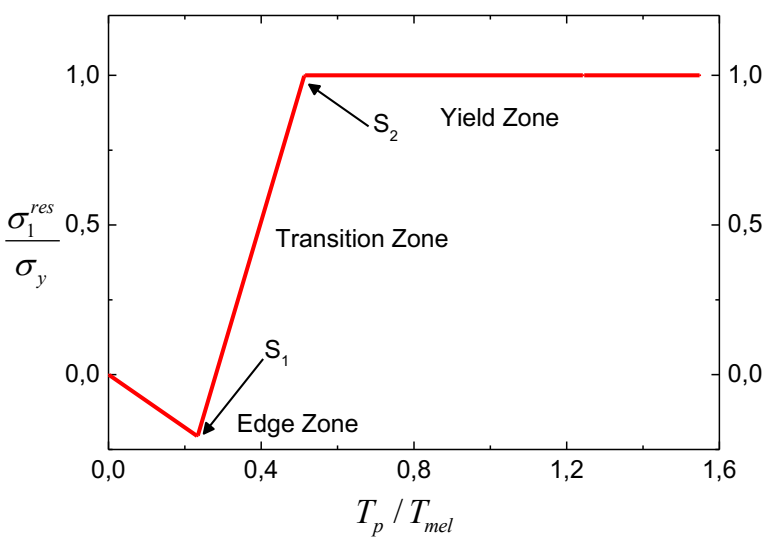

Fig. 12. Three-Segment maximum principal residual stress model.

Linear relationship in these three segments is adopted to simply link the $\sigma_{1}^{\text {res }} / \sigma_{y}$ and $T_{p} / T_{m e l}$ (Fig. 12).

The only parameters need to be identified are $\sigma_{1,1}^{r e s} / \sigma_{y}, \sigma_{1,1}^{r e s} / \sigma_{y}$ at $S_{1}$ and $S_{2}$, and $T_{1,2} / T_{m e l}$ at $S_{2}$. The formulas for each segment are presented as, 


$$
\frac{\sigma_{1}^{r e s}}{\sigma_{y}}=\left\{\begin{array}{cc}
\frac{\sigma_{1,1}^{r e s} *\left(T_{p}-T_{r}\right)}{\sigma_{y} *\left(T_{1,1}-T_{r}\right)} & \left(T_{r} \leq T_{p} \leq T_{1,1} ; 0^{\circ} \leq \theta \leq 90^{\circ}\right) \\
1-\frac{\left(T_{1,2}-T_{P}\right)}{\left(T_{1,1}-T_{1,2}\right)} *\left(1-\frac{\sigma_{1.2}^{r e s}}{\sigma_{y}}\right) & \left(T_{1,1} \leq T_{p} \leq T_{1,2} ; 0^{\circ} \leq \theta \leq 90^{\circ}\right) \\
1 & \left(T_{p} \geq T_{1,2} ; 0^{\circ} \leq \theta \leq 90^{\circ}\right)
\end{array}\right.
$$

Fig. 13 shows the evolution of $\sigma_{1,1}^{r e s} / \sigma_{y}$ as a function of the angle $\theta$ and a second-order function is applied to establish an empirical relationship,

$$
\frac{\sigma_{1,1}^{r e s}}{\sigma_{y}}=1.0 \times e^{-4} \theta^{2}-1.80 \times e^{-2} \theta+5.63 \times e^{-1}
$$

As mentioned previous, $T_{1,1} / T_{m e l}$ is equal to 0.23 , it means that the peak nodal temperature of the first critical points $S_{1}$ is constant. Hence, the distances from $S_{1}$ to center point are almost same according to section 5. For the same distance to the point heat source, close to the free surface, the material will deform more easily under the thermal load resulting in high residual plastic strain when cool down to the room temperature. A higher $\sigma_{1,1}^{\text {res }} / \sigma_{y}$ can be expected.

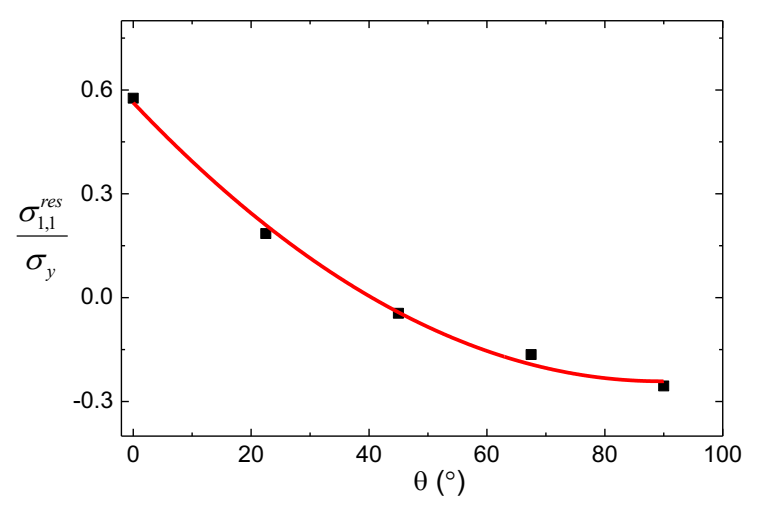

Fig. 13. Normalized first critical maximum principal residual stress $\sigma_{1,1}^{\text {res }} / \sigma_{y}$ versus angle $\theta$.

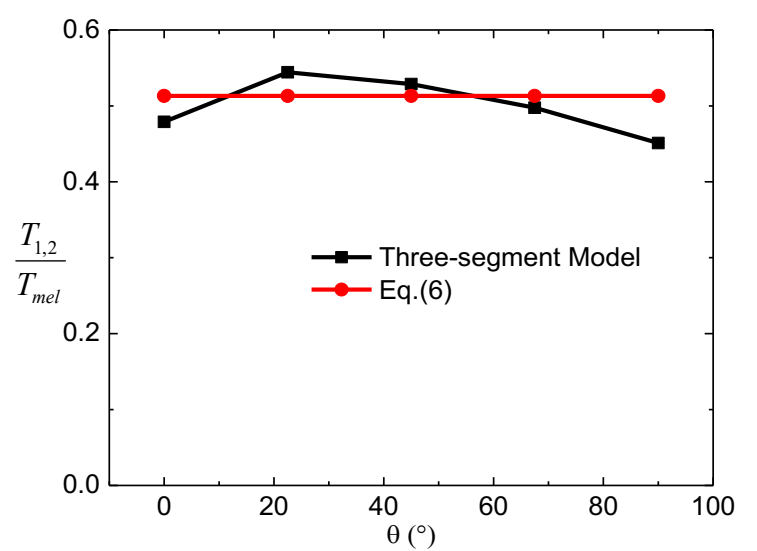

Fig. 14. Normalized second critical temperature $T_{1,2} / T_{\text {mel }}$ versus angle $\theta$. 
The peak nodal temperatures $T_{1,2}$ at $S_{2}$ are extracted and $T_{1,2} / T_{m e l}$ is plotted in Fig. 14. As can be seen, the $T_{1,2} / T_{m e l}$ close to 0.51 at different angles. Similar to $T_{e, 2}$ in three-segment equivalent residual stress model, it is more convenient to obtain $T_{1,2} / T_{m e l}$ with known yield stress by Eq. (6).

\section{Peak nodal temperature distribution model}

As discussed above, $\sigma_{e}^{r e s}$ and $\sigma_{1}^{r e s}$ are influenced by $T_{p}$ and its spatial position for a given heat input. However, the influence of $q$ on $T_{p}$ is unknown. For this concern, the heat source model introduced in section 3 is used, with the value of $q$ varying from $2.5 \times 10^{7} \mathrm{~W} / \mathrm{m}^{2}$ to $4.5 \times 10^{7} \mathrm{~W} / \mathrm{m}^{2} . T_{p}$ of the nodes in a given direction as a function of their distance $d$ to the point heat source center is presented in Fig. 15 (a) -19 (a).

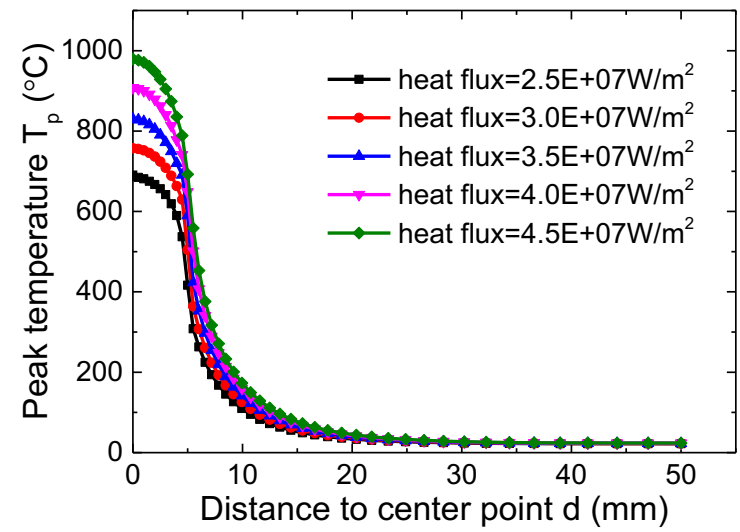

(a)

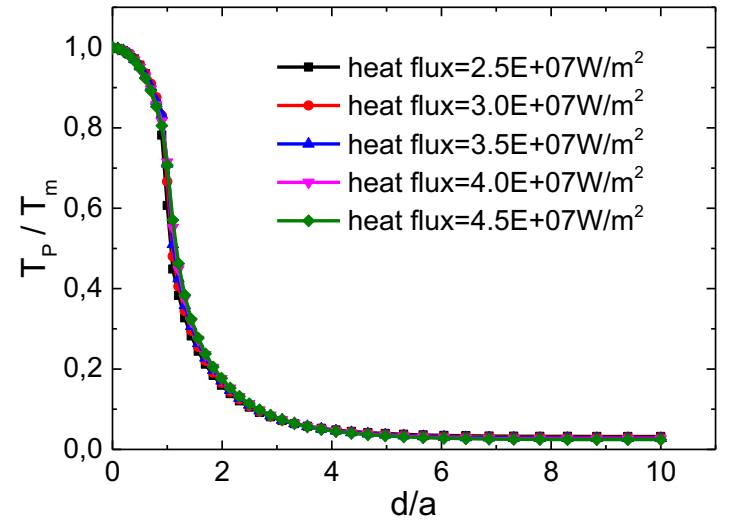

(b)

Fig. 15. (a) Peak nodal temperature distribution in the direction $\theta=0^{\circ}$; (b) Normalized $T_{p}$ versus Normalized $d$ of Fig. 15 (a).

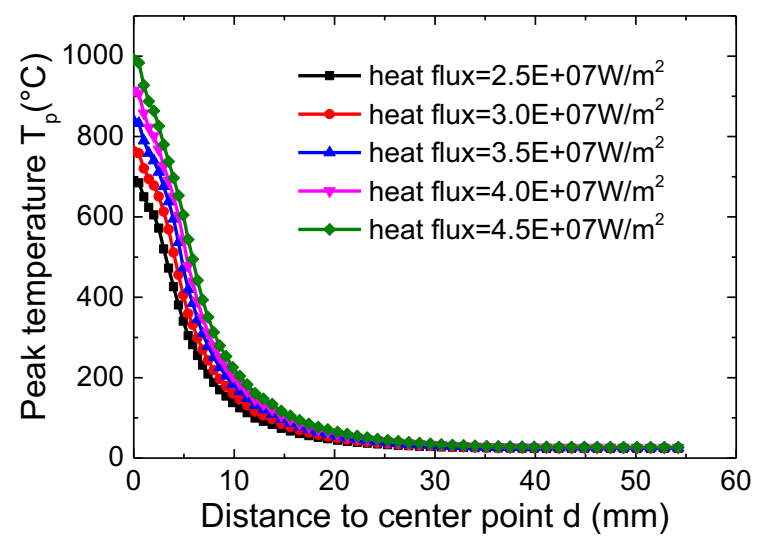

(a)

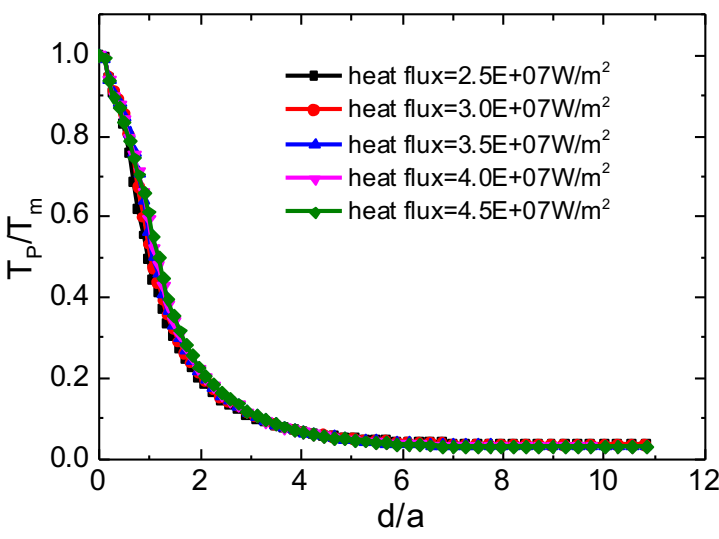

(b)

Fig. 16. (a) Peak nodal temperature distribution in the direction $\theta=22.5^{\circ}$; (b) Normalized $T_{p}$ versus Normalized $d$ of Fig. 16 (a). 


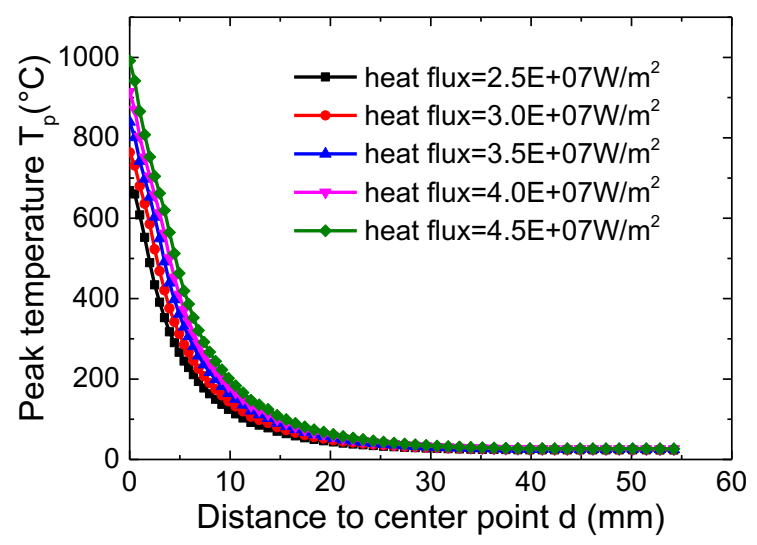

(a)

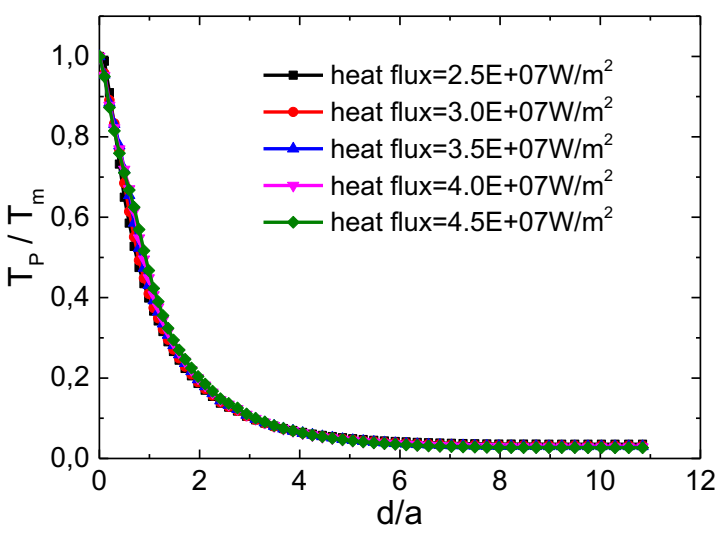

(b)

Fig. 17. (a) Peak nodal temperature distribution in the direction $\theta=45^{\circ}$; (b) Normalized $T_{p}$ versus Normalized $d$ of Fig. 17 (a).

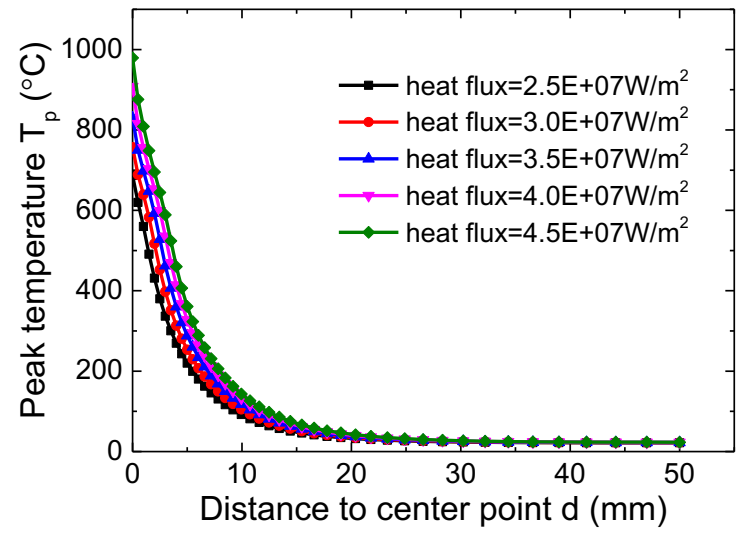

(a)

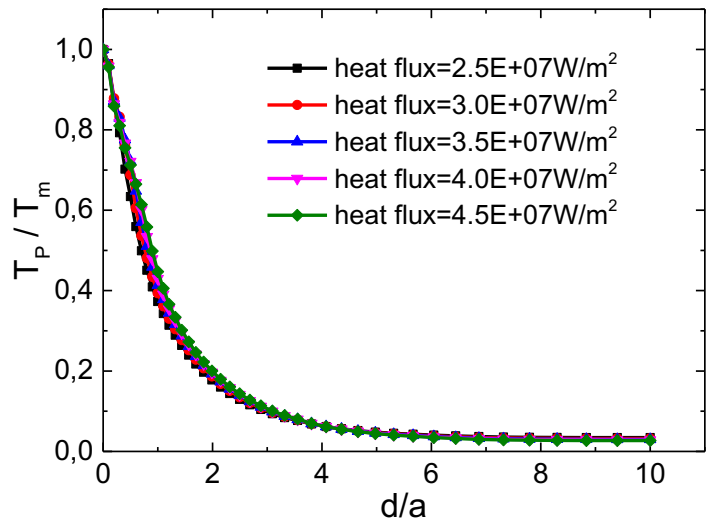

(b)

Fig. 18. (a) Peak nodal temperature distribution in the direction $\theta=67.5^{\circ}$; (b) Normalized $T_{p}$ versus Normalized $d$ of Fig. 18 (a).

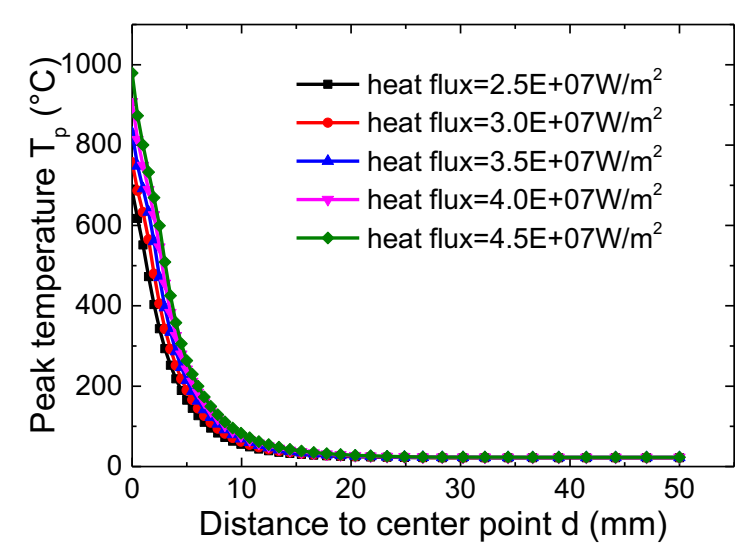

(a)

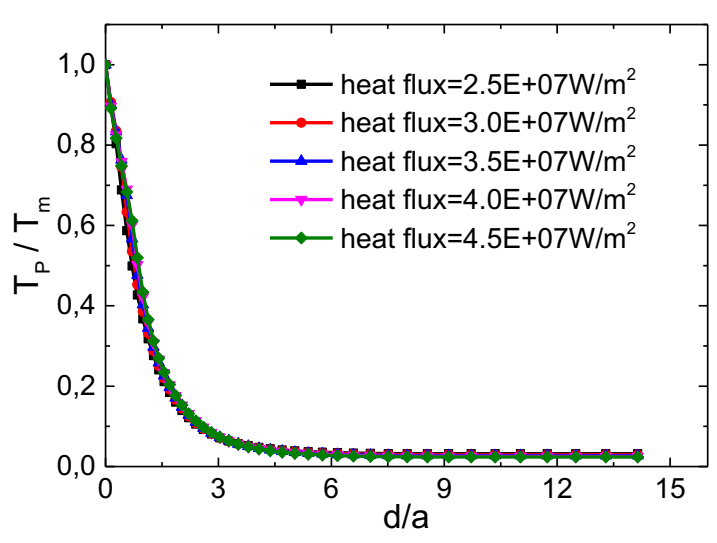

(b)

Fig. 19. (a) Peak nodal temperature distribution in the direction $\theta=90^{\circ}$; (b) Normalized $T_{p}$ versus 
For $\theta=0^{\circ}$ in Fig. 15 (a), for a node at the same position, higher heat flux yields higher peak nodal temperature, as expected. It is interesting to notice that all the curves in Fig. 15 (a) show a similar trend: $T_{p}$ decreases gradually as $d$ increases. It can also be observed that $T_{m}$ in the whole model occurs at the point heat source center. Take $T_{m}$ as a reference, all the data on the same curve is then normalized by $T_{m}$, while $d$ is normalized by the radius of the heat source $a$. The results are displayed in Fig. 15 (b). Interestingly, the normalized curves collapse almost into one. Same behavior of the $T_{p}-d$ curves and $T_{p} / T_{m}-d / a$ is also observed in Fig. $16-19$ with the angle ranging from $22.5^{\circ}$ to $90^{\circ}$.

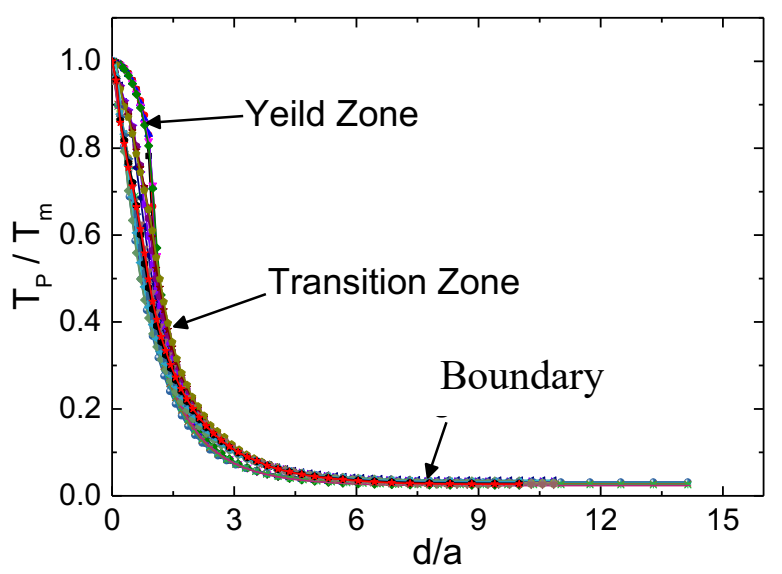

Fig. 20. Normalized peak nodal temperature $T_{p} / T_{m}$ versus Normalized distance $d / a$ with

$$
\theta=0^{\circ}, 22.5^{\circ}, 45^{\circ}, 67.5^{\circ}, 90^{\circ} \text {. }
$$

The normalized curves in Fig. 16 (b) - Fig. 19 (b) are replotted in Fig. 20. As can be seen, the normalized curves from different directions distribute very close to each other, though there are deviations when the temperature is relatively high $\left(T_{p} / T_{m} \geq 0.6\right)$. The curves in Fig. 20 is then fitted by a polynomial function,

$$
\frac{T_{P}}{T_{m}}=-4.009 \times 10^{-5}\left(\frac{d}{a}\right)^{5}+1.689 \times 10^{-3}\left(\frac{d}{a}\right)^{4}-2.709 \times 10^{-2}\left(\frac{d}{a}\right)^{3}+2.064 \times 10^{-1}\left(\frac{d}{a}\right)^{2}-0.748\left(\frac{d}{a}\right)+1.078
$$

The fitted function only depends on $T_{m}$ and $d / a$. The fitted curve can be divided into the same three segments, namely, the yield zone, the transition zone, and the boundary zone, according to the discussions in section 3 . When $T_{p} / T_{m} \geq 0.6 T_{P}$ is larger than $330^{\circ} \mathrm{C}$ and corresponds to the yield zone where the residual stress is almost constant and equal to the yield stress. Therefore, the effect of the angle can be neglected, and the error introduced due to the fitting is acceptable. In this work, the radius of the point heat source is $5 \mathrm{~mm}$. However, the fitted function is suitable for any spot size because the radius of the point heat source is normalized by the radius of the axisymmetric model in the function.

$T_{m}$ used in Fig. 16 (b) - Fig. 19 (b) are plotted against the values of $q$ and are presented in Fig. 21. A linear fitting is then applied to link the $q$ and $T_{m}$, 


$$
T_{m}=1.5113 \times 10^{-7} q+310.69
$$

It should be noted that Eq. (9) can be applied when $d / a$ is less than 15 and the Eq. (10) can be used when $T_{m}$ is larger than the melting temperature. Another point to note is that the peak nodal temperature model can not be applied when the heating time is far away from $2 \mathrm{~s}$ corresponding to the deposition speed is far away from $5 \mathrm{~mm} / \mathrm{s}$. However, the three-segment residual stress model is still applicable to predict residual stress, if the peak nodal temperature can be obtained by other methods.

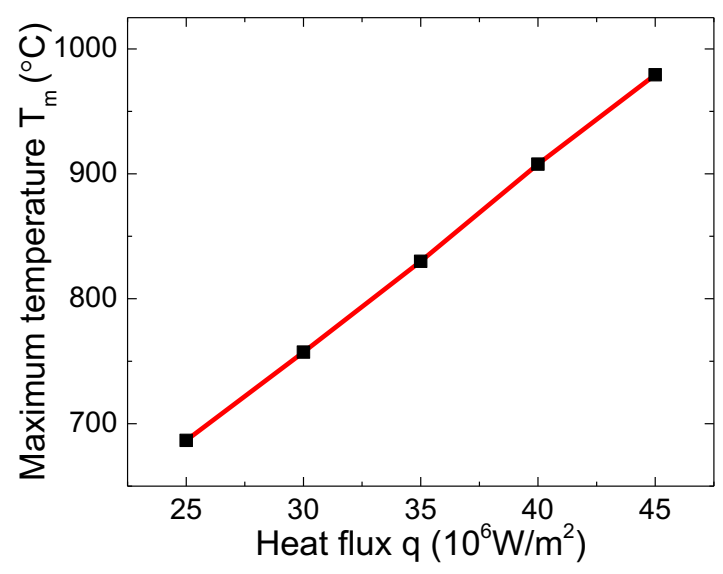

Fig. 21. Maximum temperature $T_{m}$ versus heat flux $q$.

Now, $T_{p}$ can be obtained by Eq. (9) - (10). By combining Eq. (4) - (6) and Eq. (9) - (10), $\sigma_{e}^{\text {res }}$ of a given material point can be predicted. Similarly, with Eq. (7) - (10), $\sigma_{1}^{\text {res }}$ can also be achieved. Therefore, $\sigma_{e}^{\text {res }}$ and $\sigma_{1}^{r e s}$ of a given material point can be expressed in a general form:

$$
\sigma_{\text {residual }}=f\left(q, \frac{d}{a}, \theta\right)
$$

\section{Verification and discussion}

\subsection{Verification of three-segment model in the case of point heat source}

To verify the three-segment equivalent residual stress model, the point heat source case with a heat flux $3.7 \times 10^{7} \mathrm{~W} / \mathrm{m}^{2}$ has been analyzed numerically. The geometry and parameters used in the three-segment model are the same mentioned in sections $3-4$. 
The equivalent residual stress distribution in different directions calculated by the three-segment model and from numerical analysis are compared in Fig. 22. An overall satisfactory agreement can be seen in Fig. 22 (a) - (c), especially for the results in the angle $\theta=45^{\circ}$ displayed in Fig. 22 (b). In these figures, the $\sigma_{e}^{r e s}$ calculated by the three-segment model is higher than the corresponding numerical results when $d$ is very small. The average errors are $45.0 \mathrm{MPa}, 15.6 \mathrm{MPa}$ and $13.2 \mathrm{MPa}$, which are $18.5 \%, 6.4 \%$ and $5.4 \%$ of yield stress respectively in different directions $\left(\theta=0^{\circ}, 45^{\circ}, 90^{\circ}\right)$. The reason is that the release zone is merged into the yield zone as mentioned previously. Hence, $\sigma_{e}^{r e s}$ is constant and equal to the yield stress for the materials close to the point heat source. Since the release zone is very small, the errors can be neglected. For the rest nodes, the average errors are $13.0 \mathrm{MPa}, 12.4 \mathrm{MPa}$ and $73.0 \mathrm{MPa}$, which are $5.3 \%, 5.1 \%$, and $30.0 \%$ of yield stress respectively in different directions $\left(\theta=0^{\circ}, 45^{\circ}, 90^{\circ}\right)$.

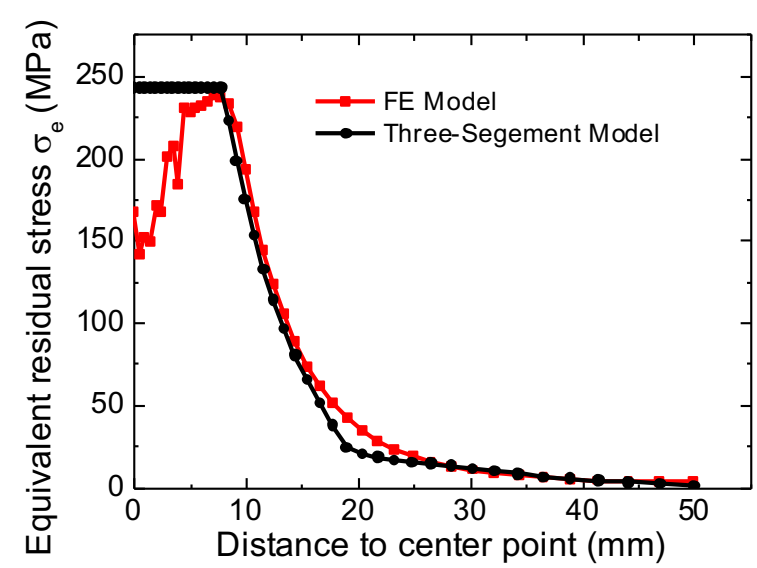

(a)

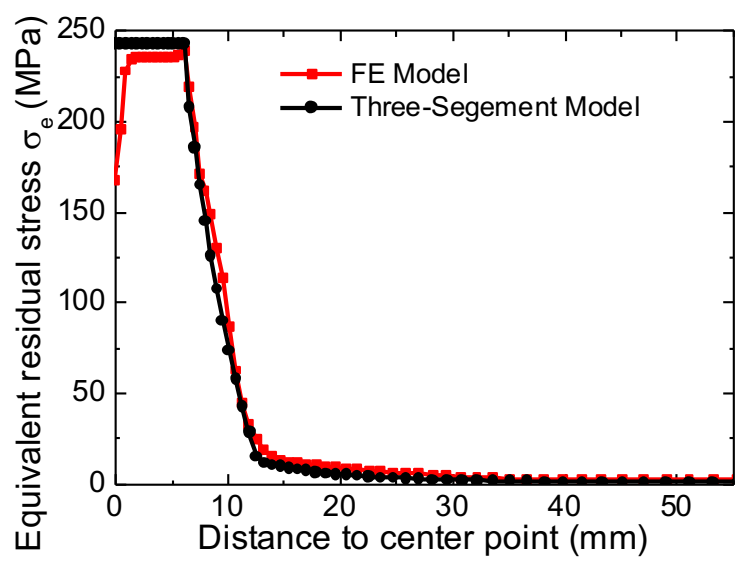

(b)

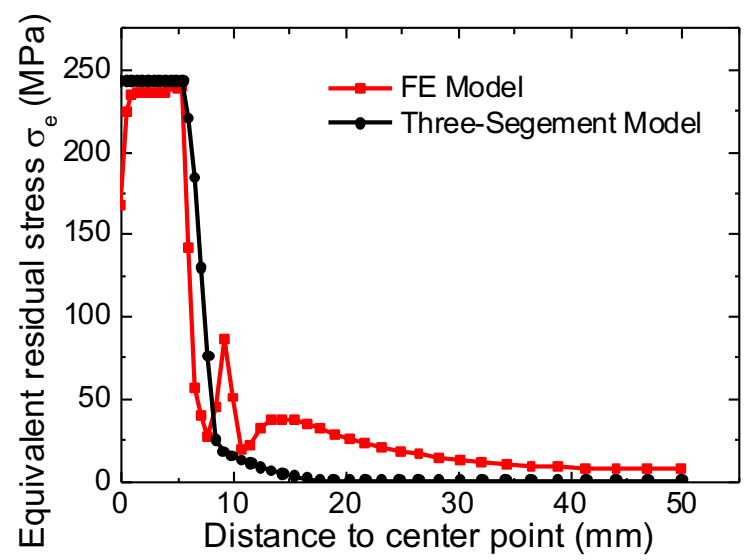

(c)

Fig. 22. Verification of the three-segment model and FE model (a) $\theta=0^{\circ}$; (b) $\theta=45^{\circ}$; (c) $\theta=90^{\circ}$.

Similar observations can be found for the comparison of the maximum principal residual stress obtained from the three-segment model and from numerical modeling, as presented in Fig. 23. For the yield zone, the average errors are $32.4 \mathrm{MPa}, 22.4 \mathrm{MPa}$ and $28.6 \mathrm{MPa}$, which are $13.3 \%, 9.7 \%$, and $11.8 \%$ respectively in different directions $\left(\theta=0^{\circ}, 45^{\circ}, 90^{\circ}\right)$. For the rest nodes, the average errors are $23 \mathrm{MPa}$, 33.5 $\mathrm{MPa}, 62 \mathrm{MPa}$, which are $9.5 \%, 13.8 \%, 25.6 \%$ of yield stress respectively in different 
directions $\left(\theta=0^{\circ}, 45^{\circ}, 90^{\circ}\right)$. For $\theta=90^{\circ}$, the errors in Fig. 21. and Fig. 22. are large, which are induced by the simplification of the temperature distribution model and linear fitting of the three-segment model.

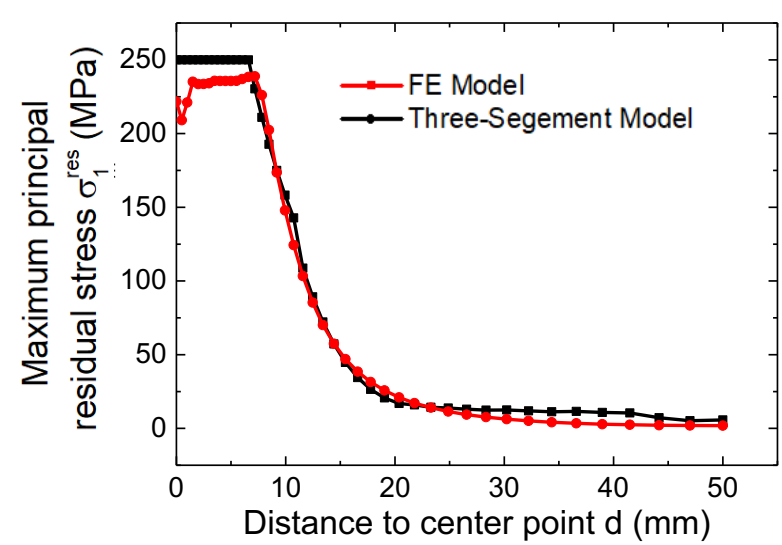

(a)

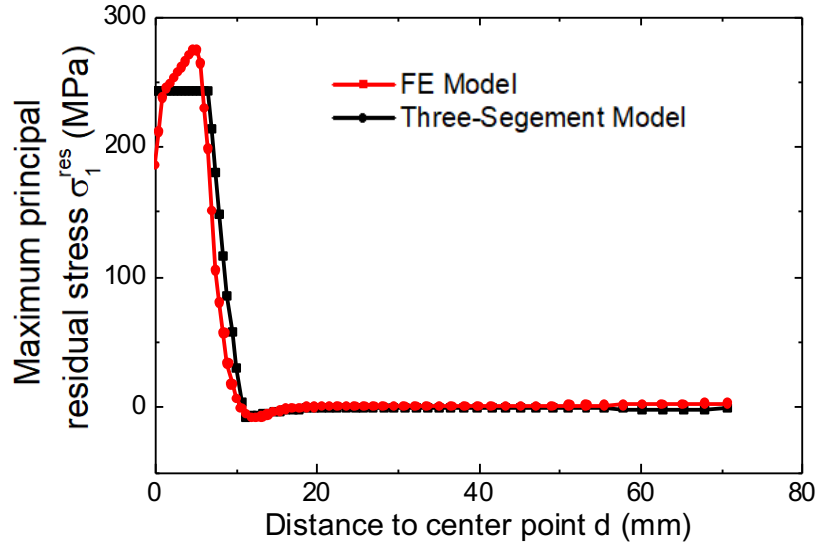

(b)

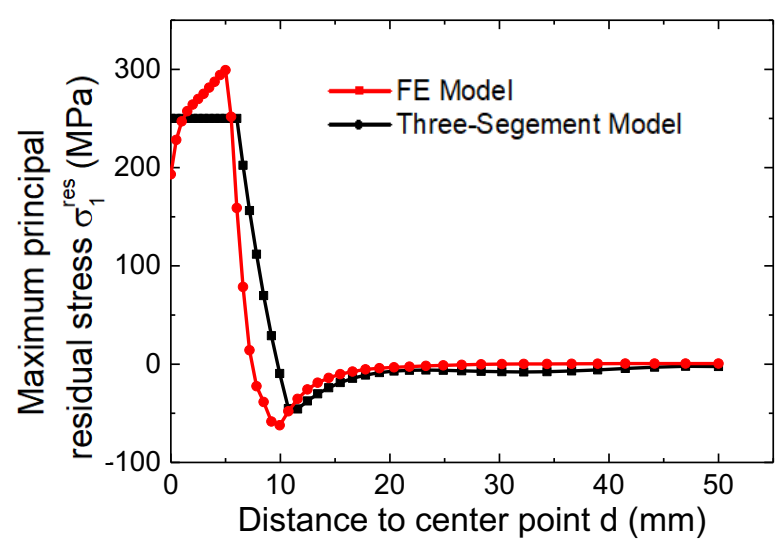

(c)

Fig. 23. Verification of three-segment method and FE model with (a) $\theta=0^{\circ}$; (b) $\theta=45^{\circ}$; (c) $\theta=90^{\circ}$.

\subsection{Verification of the three-segment model in the case of AM}

The three-segment model has also been verified by a numerical case study: additive manufactured component with thin-walled structure. The material used for the substrate and the material feedstock in this section is the same as introduced before (AA2319). Ten deposition layers are considered. The deposition speed is $600 \mathrm{~mm} / \mathrm{min}$. For the AM modeling, it is a sequentially coupled transient finite element model with a moving heat source: double ellipsoid heat source. The element birth technique is used for simulating the addition of new material, which gradually increased the stiffness of the component during the deposition process[29]. The height and width of the layer are $2.235 \mathrm{~mm}$ and 6.28 $\mathrm{mm}$, respectively. The bottom of the substrate is fixed while other surfaces are free. The geometry of the substrate is $300 \mathrm{~mm}(X) \times 60 \mathrm{~mm}(Y) \times 19 \mathrm{~mm}(Z)$.

For a line heat source, Okerblom [100] recognized that the thermal gradients transverse to the deposition direction are typically steep, whereas the gradients parallel to the weld are relatively gradual. This fact 
suggested a simple treatment for longitudinal contraction, in terms of a transverse plane strain slice, which is passed through the quasi-stationary temperature field.

In this work, similar to the simplifications of Okerblom's welding model, the heat propagates only perpendicular to the deposition direction. Hence, line heat source can be regarded as point heat source in the direction perpendicular to the deposition direction (the direction of the X-axis) while the length is about ten times of the width of the deposition layer. The three-segment model based on the point heat source model can be generalized to the AM thin-walled structure in the direction of the X-axis.

Fig. 24 (a) and (b) show the $\sigma_{e}^{\text {res }}$ and $\sigma_{1}^{\text {res }}$ distribution on the printed AM model. The residual stresses compared here are obtained from the substrate, as outlined in Fig. 24 by the red line, after finishing printing of the whole layers. Since it is more convenient to obtain the maximum temperature of the substrate in reality instead of the heat flux. The maximum temperature of $664{ }^{\circ} \mathrm{C}$ is directly output to calculate the peak nodal temperatures of the nodes along the red line with the peak nodal temperature distribution model.

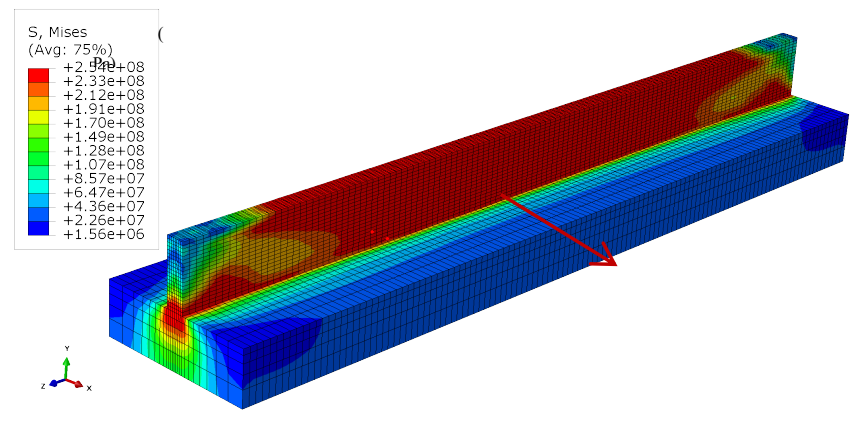

(a)

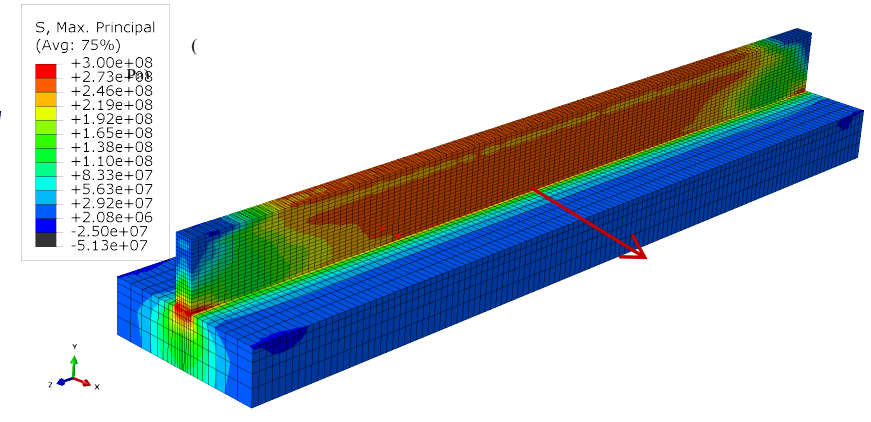

(b)

Fig. 24. The residual stress distribution in the AM model (a) Equivalent residual stress distribution; (b) Maximum principal residual stress distribution.

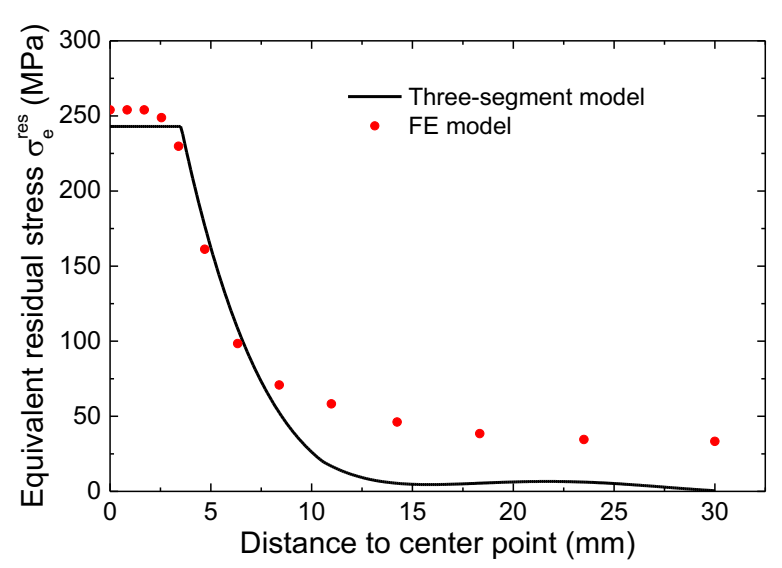

(a)

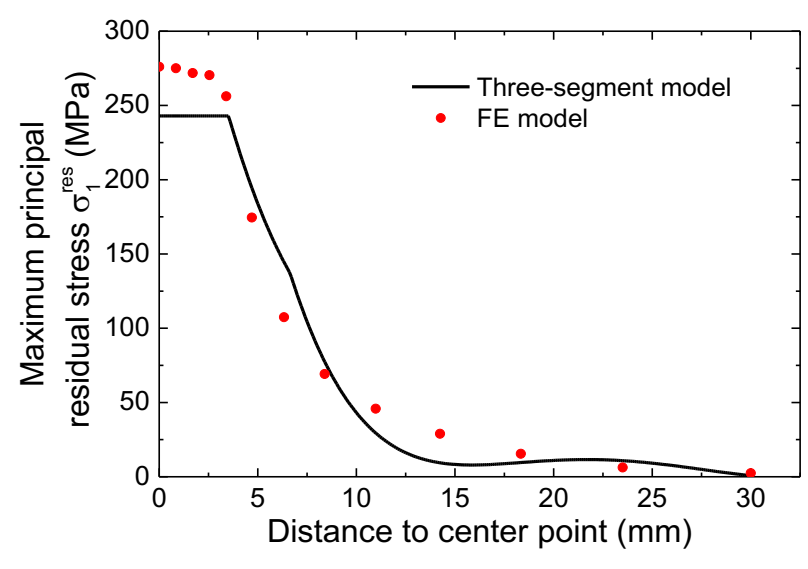

(b)

Fig. 25. Verification of the proposed three-segment model and FE model (a) Equivalent residual stress distribution (b) Maximum principal residual stress distribution. 
The $\sigma_{e}^{r e s}$ and $\sigma_{1}^{r e s}$ along the red line, calculated by the three-segment model and obtained from the FE method, are compared and presented in Fig. 25, which shows a satisfactory agreement. The threesegment model can capture the two critical points precisely. For $\sigma_{e}^{\text {res }}$, the average errors are $10.4 \mathrm{MPa}$, 14.6 $\mathrm{MPa}$ and $35.3 \mathrm{MPa}$, which are $4.3 \%, 6.0 \%$, and $14.5 \%$ of yield stress respectively in yield zone, transition zone, and boundary zone. For $\sigma_{1}^{\text {res }}$, the average errors are $26.9 \mathrm{MPa}, 21.6 \mathrm{MPa}$ and $7.9 \mathrm{MPa}$, which are $11.1 \%, 8.9 \%$ and $3.4 \%$ of yield stress respectively in yield zone, transition zone, and boundary zone. There are deviations when the material is relatively far from the printed layer. These errors may be introduced due to the thin substrate thickness and the boundary condition. The approach derived from the point heat source model can be applied to estimate the residual stress of the substrate in AM and greatly improves the computational efficiency, compared to other methods.

For welding or additive manufacturing, as long as that the length of the component in at least one direction perpendicular to the deposition direction is ten times larger than the radius of heat source, the manufactured component can be regarded as a model characterized by point heat source in that direction and the three-segment model can predict the residual stress along that direction. However, the limitation of the proposed method in additive manufacturing is that it can only be applied to estimate the residual stresses of the substrate of the thin-walled structure component. Because, for moving heat source, only single line heat source can be regarded as a superposition of instantaneous point heat source in the direction perpendicular to the deposition.

\section{Conclusions}

In this work, a three-segment model is proposed to estimate the equivalent and the maximum principal residual stress in manufacturing, based on the analyses of a point heat source. A three-segment residual stress model and a simplified peak nodal temperature distribution model are first proposed. In these two models, the residual stress distribution can be divided into yield zone, transition zone and boundary zone by peak nodal temperature and the peak nodal temperature only depends on the heat flux and the distance to the point heat source center. Hence, the final functions based on these two models indicate that the residual stress of a material point depends on the heat flux, node spatial position and intrinsic properties of a material. For the point heat source model and the numerical AM model, the results generated by the three-segment model show good agreement with FE results. It should be noted that the exact functions derived in this work can only be accurate for the AA 2319, however, this methodology can equally be generalized to other materials that show no phase transformations (e.g. copper, austenitic stainless steels, etc.). The three-segment model can also be applied to many manufacturing processes characterized by the point heat source model, such as the welding, laser heat treatment, etc. The limitation of the proposed method in additive manufacturing is that it can only be applied to estimate the residual stresses of the 
substrate of the thin-walled structure component. Compared with other methods, the three-segment model with simple calculation process is extremely efficient, which can reduce the calculating cost significantly, especially for large AM components and can thus be readily used in an industrial context.

\section{Acknowledgment}

The Chinese Scholarship Council is greatly acknowledged for financial support. The authors wish to thank the Research Council of Norway through the BIA Program, Contract No. 269558/O20 and the Academy of Finland (311934) for funding.

\section{Reference}

1. Kranz J, Herzog D, Emmelmann C (2015) Design guidelines for laser additive manufacturing of lightweight structures in TiAl6V4. Journal of Laser Applications 27 (S1):S14001. doi:10.2351/1.4885235

2. Carroll BE, Palmer TA, Beese AM (2015) Anisotropic tensile behavior of Ti-6Al-4V components fabricated with directed energy deposition additive manufacturing. Acta Materialia 87:309-320. doi:10.1016/j.actamat.2014.12.054

3. Brandl E, Palm F, Michailov V, Viehweger B, Leyens C (2011) Mechanical properties of additive manufactured titanium (Ti-6Al-4V) blocks deposited by a solid-state laser and wire. Materials \& Design 32 (10):4665-4675. doi:10.1016/j.matdes.2011.06.062

4. Mercelis P KJ (2006) Residual stresses in selective laser sintering and selective laser melting. Rapid Prototyp $12((5)): 254-265$

5. Gary K. Lewis ES (2000) Practical considerations and capabilities for laser assisted direct metal deposition. Materials and Design 21:417]423

6. Deng D (2009) FEM prediction of welding residual stress and distortion in carbon steel considering phase transformation effects. Materials \& Design 30 (2):359-366. doi:10.1016/j.matdes.2008.04.052

7. Labudovic M HD, Kovacevic R (2003) A three dimensional model for direct laser metal powder deposition and rapid prototyping. Journal of Materials Science 38 ((1)):35-49

8. Smith J, Xiong W, Yan W, Lin S, Cheng P, Kafka OL, Wagner GJ, Cao J, Liu WK (2016) Linking process, structure, property, and performance for metal-based additive manufacturing: computational approaches with experimental support. Computational Mechanics 57 (4):583-610. doi:10.1007/s00466015-1240-4

9. J.L. Songa ALD, M.H. Jacobsa, J. Brooksb, I. Bedenc (2002) Coupled thermo-mechanical finiteelement modelling of hot ring rolling process. Journal of Materials Processing Technology 121:332-340

10. Zhang Z, Zhang HW (2007) A fully coupled thermo-mechanical model of friction stir welding. The International Journal of Advanced Manufacturing Technology 37 (3-4):279-293. doi:10.1007/s00170007-0971-6 
11. Yuan MG UY (Yuan MG, Ueda Y.) Prediction of Residual Stresses in Welded T- and 1-Joints Using Inherent Strains. Journal of Engineering Materials and Technology 118 ((2)):229-234

12. Li C, Fu CH, Guo YB, Fang FZ (2016) A multiscale modeling approach for fast prediction of part distortion in selective laser melting. Journal of Materials Processing Technology 229:703-712. doi:10.1016/j.jmatprotec.2015.10.022

13. Yang Y, Ayas C (2017) Computationally efficient thermal-mechanical modelling of selective laser melting. 1892:040005. doi:10.1063/1.5008031

14. Mukherjee T, Manvatkar V, De A, DebRoy T (2017) Mitigation of thermal distortion during additive manufacturing. Scripta Materialia 127:79-83. doi:10.1016/j.scriptamat.2016.09.001

15. Mukherjee T, Zuback JS, De A, DebRoy T (2016) Printability of alloys for additive manufacturing. Sci Rep 6:19717. doi:10.1038/srep19717

16. Cheng W (2005) In-plane shrinkage strains and their effects on welding distortion in thin-wall structures. The Ohio State University, Ohio

17. Camilleri D, Comlekci T, Gray TGF (2005) Computational prediction of out-of-plane welding distortion and experimental investigation. The Journal of Strain Analysis for Engineering Design 40 (2):161-176. doi:10.1243/030932405x7809

18. Ding J, Colegrove P, Mehnen J, Williams S, Wang F, Almeida PS (2013) A computationally efficient finite element model of wire and arc additive manufacture. The International Journal of Advanced Manufacturing Technology 70 (1-4):227-236. doi:10.1007/s00170-013-5261-x

19. Radaj D (1992 ) Heat Effects of Welding: Temperature Field, Residual Stress, Distortion. Springer Science \& Business Media,

20. C. K. Chao FMC, M. H. Shen (2006) Green's Functions for a Point Heat Source in Circularly Cylindrical Layered Media. Journal of Thermal Stresses 29:809-847. doi:10.1080/01495730600705430

21. Pratik P. Shukla PTS, Colin J (2014) Laser shock peening and mechanical shot peening processes applicable for the surface treatment of technical grade ceramics: A review. Journal of Engineering Manufacture 228 ((5)):639-652. doi:10.1177/0954405413507250

22. Hasebe KYN (1999) Green's Function for a Heat Source in an Infinite Region With an Arbitrary Shaped Hole. Journal of Applied Mechanics 66:204-210

23. Nazemi N (2015) Identification of the mechanical properties in the heat-affected zone of aluminum welded structures. University of Windsor, Canada

24. Meco SAM (2016) Joining of steel to aluminium alloys for advanced structural applications. Cranfield University, United Kingdom

25. Ding J (2012) Thermo-mechanical Analysis of Wire and Arc Additive Manufacturing Process. Cranfield University, United Kingdom

26. Michaleris P, Feng, Z., and Campbell, G., (1997) Evaluation of 2D and 3D FEA Models for Predicting Residual Stress and Distortion. ASME PVP- Approximate Methods in the Design and Analysis of Pressure Vessels and Piping Components 347:91-102 
27. Martukanitz R, Michaleris P, Palmer T, DebRoy T, Liu Z-K, Otis R, Heo TW, Chen L-Q (2014) Toward an integrated computational system for describing the additive manufacturing process for metallic materials. Additive Manufacturing 1-4:52-63. doi:10.1016/j.addma.2014.09.002

28. DebRoy T, Wei HL, Zuback JS, Mukherjee T, Elmer JW, Milewski JO, Beese AM, Wilson-Heid A, De A, Zhang W (2018) Additive manufacturing of metallic components - Process, structure and properties. Progress in Materials Science 92:112-224. doi:10.1016/j.pmatsci.2017.10.001

29. Y. Ueda KF, K. Nakacho and S. Endo (1975) A new measuring method of residual stresses with the aid of finite element method and reliability of estimated values. Soc Nav Archit:499-507 30. Okerblom NO (1958) The Calculations of Deformations of Welded Metal Structures. Her Majesty's Stationery Office, Londo 